TU-809

\title{
Axionic Mirage Mediation
}

\author{
Shuntaro Nakamura, Ken-ichi Okumura and Masahiro Yamaguchi \\ Department of Physics, Tohoku University, Sendai, 980-8578, Japan
}

\begin{abstract}
Although the mirage mediation is one of the most plausible mediation mechanisms of supersymmetry breaking, it suffers from two crucial problems. One is the $\mu-/ B \mu$-problem and the second is the cosmological one. The former stems from the fact that the $B$ parameter tends to be comparable with the gravitino mass, which is two order of magnitude larger than the other soft masses. The latter problem is caused by the decay of the modulus whose branching ratio into the gravitino pair is sizable. In this paper, we propose a model of mirage mediation, in which Peccei-Quinn symmetry is incorporated. In this axionic mirage mediation, it is shown that the Peccei-Quinn symmetry breaking scale is dynamically determined around $10^{10} \mathrm{GeV}$ to $10^{12} \mathrm{GeV}$ due to the supersymmetry breaking effects, and the $\mu$-problem can be solved naturally. Furthermore, in our model, the lightest supersymmetric particle (LSP) is the axino, that is the superpartner of the axion. The overabundance of the LSPs due to decays of modulus/gravitino, which is the most serious cosmological difficulty in the mirage mediation, can be avoided if the axino is sufficiently light. The next-LSPs (NLSPs) produced by the gravitino decay eventually decay into the axino LSPs, yielding the dominant component of the axinos remaining today. It is shown that the axino with the mass of $\mathcal{O}(100) \mathrm{MeV}$ is naturally realized, which can constitute the dark matter of the Universe, with the free-streaming length of the order of $0.1 \mathrm{Mpc}$. The saxion, the real scalar component of the axion supermultiplet, can also be cosmologically harmless due to the dilution of the modulus decay. The lifetime of NLSP is relatively long, but much shorter than 1 sec., when the big-bang nucleosynthesis commences. The decay of NLSP would provide intriguing collider signatures.
\end{abstract}




\section{Introduction}

Supersymmetry (SUSY) is one of the most promising candidates for new physics beyond the standard model (SM), which solves the naturalness problem associated with the hierarchy between the electroweak scale and the Planck scale. However, since no supersymmetric particles have been discovered yet, SUSY has to be broken. In order to prevent us from arising the new dangerous phenomenological effects, flavor-changing-neutral-current (FCNC) process and SUSY $\mathrm{CP}$ violation and so on, the SUSY-breaking mediation mechanism must be the one that does not give rise to these effects. In superstring theory, one of most plausible mediation mechanisms without these effects is the moduli mediation [1]. The contribution of the modulus, $X$, to the soft masses is proportional to its auxiliary component, $F_{X} \simeq m_{3 / 2}^{2} / m_{X}$ (in the Planck unit $M_{\mathrm{Pl}}=1$ ), where $m_{3 / 2}$ and $m_{X}$ are the gravitino mass and the modulus mass, respectively. On the other hand, in 4-dimensional supergravity (SUGRA), the mediation associated with the super-Weyl anomaly, the anomaly mediation [2]-[4], also does exist, whose contribution to the soft masses is of the order of $m_{3 / 2} / 8 \pi^{2}$. Recently, KKLT [5] have proposed the interesting set-up to stabilize the modulus with the relatively heavy mass, $m_{X} \simeq 4 \pi^{2} m_{3 / 2}$, by considering some non-perturbative effects. Therefore, in this and similar set-ups, the anomaly mediation contribution is comparable with the modulus mediation one. This type of mediation mechanism is often called the mirage mediation [6]-[15].

The mirage mediation is a natural mediation mechanism and quite interesting because it can solve the tachyonic slepton problem in the pure anomaly mediation, as well as has characteristic mass spectrums [9] [10]. However, it has two crucial problems. One of them is the $\mu$ - $/ B \mu$-problem. In the discussion of ref.[10], although it is not difficult to obtain that $\mu$ is of the order of the soft masses, $B$ becomes of the order of $m_{3 / 2}$, in general, without fine-tuning of parameters. In the mirage mediation, since the gravitino mass should be $\mathcal{O}(10) \mathrm{TeV}$ in order to obtain soft masses with the electroweak scale, this is problematic.

The other problem results from cosmology. While in inflation the modulus field is shifted from the true minimum. When the Hubble parameter $H$ decreased to the order of the modulus mass, $m_{X}$, the modulus starts to oscillate around the true minimum with amplitude of order the Planck scale. The energy density of the coherent oscillation dominates that of the Universe before the modulus decay. If the modulus mass is around the electroweak scale, its decay produces immense amount of entropy after primordial nucleosynthesis and upsets the success of the bigbang nucleosynthesis (BBN). Such a cosmological catastrophe is known as the moduli problem [16]. One of the resolutions to the moduli problem is to invoke a relatively heavy modulus with mass of $10^{5} \mathrm{GeV}$ or larger. The heavy modulus then decays before the nucleosynthesis commences, which will not spoil it. Thus, KKLT set-up seems to be favored cosmologically.

However, recently, it was found that non-thermal production of the gravitino from the modulus decay is sizable, which aggravates the moduli problem [17] [18]. (See, for the case of the inflaton and the Polonyi field [19]-[22]). Such aggravation comes not only from abundance of the gravitino but also from the overclosure of the neutralinos if it is the lightest superparticle (LSP). The gravitinos with the electroweak scale mass decay into minimal supersymmetric standard model 
(MSSM) particles after or during the primordial nucleosynthesis, and hence the decay products may spoil the success of BBN. This is called the gravitino problem. It is known that when the gravitino mass is as large as $\mathcal{O}(10) \mathrm{TeV}$, there is no gravitino problem because of their sufficiently short lifetime. On the other hand, the neutralinos produced by the decay of the gravitinos are so abundant that the annihilation process is effective. However, its abundance after the annihilation exceeds the present dark matter (DM) abundance even if the neutralino LSP is the wino, whose annihilation cross section is most effective. Therefore, even if the modulus can decay before BBN, it causes the cosmological problem. This disaster may be remedied when LSP is lighter than the neutralino.

We will solve these two problems simultaneously by the axionic extension of the mirage mediation (axionic mirage mediation). In SM, the instanton effect of the quantum chromodynamics (QCD) generate the following term in the Lagrangian

$$
\mathcal{L}_{\bar{\Theta}}=\frac{g_{3}^{2}}{64 \pi^{2}} \bar{\Theta} \epsilon^{\mu \nu \rho \sigma} G_{\mu \nu}^{a} G_{\rho \sigma}^{a},
$$

where $g_{3}$ is the strong coupling constant, $G_{\mu \nu}^{a}$ the field strength of the gluon and $\bar{\Theta}=\Theta+$ $\arg (\operatorname{det} \mathcal{M})$ with the quark mass matrix $\mathcal{M}$. From the present experiment of the electric dipole moment of the neutron, $\bar{\Theta}$ has to be smaller than $10^{-9}$. In SM, however, there are no reasons that $\bar{\Theta}$ should be so small, because it just a parameter of the Lagrangian. This is well known as the strong $\mathrm{CP}$ problem. One attractive solution to this problem is to introduce an anomalous global $\mathrm{U}(1)$ symmetry, called Peccei-Quinn (PQ) symmetry, $\mathrm{U}(1)_{\mathrm{PQ}}[23]$. When the $\mathrm{U}(1)_{\mathrm{PQ}}$ is spontaneously broken, a pseudo Nambu-Goldstone boson, called axion $a$, appears. Since the axion couples with the gluon as

$$
\mathcal{L}=\frac{g_{3}^{2}}{64 \pi^{2}}\left(\bar{\Theta}+\frac{a}{f_{a}}\right) \epsilon^{\mu \nu \rho \sigma} G_{\mu \nu}^{a} G_{\rho \sigma}^{a},
$$

where $f_{a}$ is the decay constant of the axion, the potential of the axion is minimized at $\bar{\Theta}+\langle a\rangle / f_{a}=$ 0 . Thus, PQ symmetry can solve the strong CP problem [24]-[26]. From the above argument, it seems reasonable to consider the supersymmetric axion model, which is studied by many authors [27]-[33]. In some supersymmetric axion models, the axino, which is a superpartner of the axion, can be LSP and become the DM candidate [34-[37. It is realized by introducing only a Yukawa coupling between an axion superfield and messengers in the superpotential [39]. In such a model, the axion superfield is a flat direction at the tree level, and hence the axino mass is induced by quantum corrections. Thus, the axino can become lighter than the neutralinos.

In this paper, we show that the axionic mirage mediation can solve not only the $\mu-/ B \mu$ problem but the cosmological moduli problem simultaneously. First, we briefly review the mirage mediation in section 2. In section 3, we discuss about our model and show that the PQ scale can be obtained within, so-called, the axion window by stabilizing the axion superfield. Throughout this paper, we will focus on the case where the axion superfield is stabilized at $10^{10} \mathrm{GeV}$. The $\mu$ problem is discussed in section 4. In this section, we also find that there is no SUSY CP problem 
in our model. The cosmological implication is devoted to section 5 and 6 . Since the modulus decay releases a huge amount of entropy, unwanted particles existed before is diluted away, and thus we discuss only after the modulus decay. Although the modulus produces a number of gravitinos, it will cause no cosmological problem because the next LSPs (NLSP) produced by the gravitinos decay into axino LSPs. We consider the cases where NLSP is the bino, the higgsino, the stau, the stop or the wino, respectively. By discussing several processes to produce the axino by non-thermally, we find that the axino with mass $\mathcal{O}(100) \mathrm{MeV}$ produced such a way can explain the present DM abundance in any NLSP cases, if the annihilation process of NLSPs produced by the gravitino decay is not effective. The saxion, the real scalar component of the axion superfield, would be also harmless because the modulus decay dilutes its decay products since the lifetime of the saxion is shorter than that of the modulus due to the relatively small decay constant $f_{\mathrm{PQ}} \simeq 10^{10} \mathrm{GeV}$. We briefly comment on whether the axion can constitute DM when the axion superfield is stabilized at $10^{12} \mathrm{GeV}$. Section 7 is devoted to summary. ${ }^{1}$

\section{Mirage mediation of supersymmetry breaking}

First we briefly review the mirage mediation of the SUSY breaking [6]-[14]. Let us consider the following supergravity (SUGRA) $\Omega$ function and superpotential ${ }^{2}$ :

$$
\begin{gathered}
\Omega=-3\left(X+X^{\dagger}\right)+\left(X+X^{\dagger}\right)^{q_{I}}\left|Q_{I}\right|^{2}, \\
W=W_{0}(X)+\frac{1}{6} \lambda_{I J K} Q_{I} Q_{J} Q_{K},
\end{gathered}
$$

where $X$ and $Q_{I}$ represent the modulus field and the chiral matter fields, respectively. The modulus superpotential, $W_{0}(X)$, which is responsible for stabilization of moduli, arises from non-perturbative effects like gaugino condensation or stringy instanton as well as with a modulus independent term from various origin. In the KKLT construction [5], it is given by $W_{0}=$ $A \exp (-b X)+C^{3}$. The function $\Omega$ has the relation to the Kähler potential, $K$, via

$$
\Omega=-3 e^{-K / 3} \text {. }
$$

From the SUGRA $\Omega$ function and the superpotential, the chiral/gauge fields sector of the SUGRA Lagrangian can be written by using the compensator, $\Phi$,

$$
\mathcal{L}=\int d^{4} \theta \Phi^{\dagger} \Phi \Omega+\int d \theta^{2}\left[\Phi^{3} W+\frac{1}{4} f_{a}(X) \mathcal{W}^{a \alpha} \mathcal{W}_{\alpha}^{a}\right]+\text { h.c. }
$$

where $f_{a}(X)$ is the gauge kinetic function. Assuming the shift symmetry, $X \rightarrow X+i \omega$, in absence of $W_{0}(X)$, its form is restricted to $f_{a}(X)=p_{a} X+q_{a}$. After integrating out $F_{\Phi}$, the scalar part

\footnotetext{
${ }^{1}$ Introduction of a singlet field to solve the $\mu-/ B \mu$-problem and the cosmological problem was also considered in 38. The latter problem was solved by thermal inflation.

${ }^{2}$ Throughout this paper, we apply the Planck unit $M_{\mathrm{Pl}} \simeq 2.4 \times 10^{18} \mathrm{GeV}=1$ unless we explicitly mention.

${ }^{3}$ Here we consider the single modulus case only. Extention to the multi-modulus case is straightforward.
} 
recovers the conventional SUGRA potential in the Einstein frame by choosing $\langle\Phi\rangle=\left\langle e^{K / 6}\right\rangle$,

$$
V_{\mathrm{SUGRA}}=e^{K}\left(K^{I \bar{J}} D_{I} W \overline{D_{J} W}-3|W|^{2}\right)
$$

with $F_{\Phi} /\langle\Phi\rangle=K_{I} F^{I} / 3+m_{3 / 2}$. Here, a SUSY configuration, $F^{I}=-e^{K / 2} K^{I \bar{J}} \overline{D_{J} W}=0$, gives the extremum of $V_{\text {SUGRA }}$ if it exists. It is often the case that this corresponds to the minimum of the potential and ends up with the AdS vacuum, $V_{\text {SUGRA }}=-3 e^{K}|W|^{2}=-3\left|m_{3 / 2}\right|^{2}$. To achieve observed approximately Minkowski vacuum with positive cosmological constant, we introduce the uplifting potential, $V=V_{\text {SUGRA }}+V_{\text {lift }} \approx 0^{+}$. This additional SUSY breaking potential could be remnant of stringy effects like the anti-D3 brane in the original KKLT construction [5] or field theoretic "hidden sector" effects separated from the first term of $V_{\text {SUGRA }}$ (F-term uplifting) [40]-43]. Including such a modification, the initial SUSY configuration of the modulus field, $D_{X} W_{0}(X)=0$, shifts by $|\delta X| \sim M_{P l}\left(m_{3 / 2}^{2} / m_{X}^{2}\right)$ and this induces modulus SUSY breaking, $\left|F_{X} / 2 X_{R}\right| \simeq m_{3 / 2}^{2} / m_{X}$ where $X_{R}=\operatorname{Re}(X)$. It is known that the non-perturbative stabilization predicts significant enhancement of $m_{X} / m_{3 / 2}$ due to derivative of the exponential factors in $W \sim$ $m_{3 / 2}$. Thus $F_{X} / 2 X_{R}$ is considerably suppressed relative to $m_{3 / 2}$. This means that, in the visible sector, the anomaly mediated SUSY breaking of order $\left(F_{\Phi} /\langle\Phi\rangle\right) / 8 \pi^{2} \approx m_{3 / 2} / 8 \pi^{2}$ is equally important as the modulus mediated contribution. The relative phase of two sources of the SUSY breaking potentially causes the sever SUSY CP problem [44]. However, if the complex phases in $W_{0}$ can be rotated away by symmetries which are explicitly broken by $W_{0}$ (e.g. the R-symmetry and the shift symmetry), the relative phase in $F_{X} /\left(F_{\Phi} /\langle\Phi\rangle\right)$ vanishes, which is the case in the KKLT set-up [45] 9] [10]. This new class of the SUSY breaking is dubbed "mirage mediation" after a peculiar renormalization group $(\mathrm{RG})$ behavior of its mass spectrum which we will discuss later.

Any direct coupling with the source of the SUSY breaking in the Kähler metric undermines such a loop suppressed effect in the scalar sector. Thus the sequestering of the SM fields and the hidden sector is essential for the mirage mediation. Actually, it is known that naive geometrical separation only does not guarantee the sequestering in string theory [47] [48]. However, strongly warped geometry, which is the heart of the original KKLT proposal, fulfills this requirement [5] [8] [46] [49] [50].

In literature the following constant is defined to parametrize the relative strength of the two SUSY breaking contributions,

$$
\alpha \equiv \frac{m_{3 / 2}}{\left(F_{X} / 2 X_{R}\right) \ln \left(M_{P l} / m_{3 / 2}\right)} \approx \frac{m_{3 / 2}}{4 \pi^{2}\left(F_{X} / 2 X_{R}\right)} .
$$

The KKLT set-up with the uplifting potential generated by the anti-D3 brane on the tip of the warped throat predicts $\alpha=1+\mathcal{O}\left(1 / 4 \pi^{2}\right)$, while there are variety of proposals which lead to different values of $\alpha=\mathcal{O}(1)$ [6] [10] [51] [43].

The soft SUSY breaking terms of the visible fields are parametrized as,

$$
\mathcal{L}=-\frac{1}{2} m_{\lambda_{a}} \bar{\lambda}_{a} \lambda_{a}-m_{I}^{2}\left|\tilde{\phi}_{I}\right|^{2}+A_{I J K} Y_{I J K} \tilde{\phi}_{I} \tilde{\phi}_{J} \tilde{\phi}_{K}+\text { h.c. },
$$


where $\lambda_{a}$ denotes the gaugino and $\tilde{\phi}_{I}$ is the scalar component of $Q_{I}$ in canonical normalization, while $Y_{I J K} \equiv \lambda_{I J K} / \sqrt{\left(X+X^{\dagger}\right)^{q_{I}+q_{J}+q_{K}}}$ represents the canonical Yukawa coupling constant. We perform the redefinition of the matter fields, $\hat{Q}_{I} \equiv \Phi Q_{I}$ to identify the effect of the anomaly mediation,

$$
\begin{aligned}
\mathcal{L}= & \int d^{4} \theta\left[-3\left(X+X^{\dagger}\right)|\Phi|^{2}+\left(X+X^{\dagger}\right)^{q_{I}}\left|\hat{Q}_{I}\right|^{2}\right] \\
& +\int d^{2} \theta\left[\Phi^{3} W\left(X, \hat{Q}_{I} / \Phi\right)+\frac{1}{4} f_{a}(X) \mathcal{W}^{a \alpha} \mathcal{W}_{\alpha}^{a}\right]+\text { h.c. }
\end{aligned}
$$

It is straightforward to derive the canonical gaugino mass from the above gauge kinetic function,

$$
m_{\lambda_{a}}=F_{X} \partial_{X} \ln \operatorname{Re}\left(f_{a}(X)\right) \equiv c_{\lambda_{a}} \frac{F_{X}}{2 X_{R}},
$$

while the other soft SUSY breaking terms are read off by substituting the equation of motion for the $F$-component of the light fields, $\hat{Q}_{I}$, into the above Lagrangian,

$$
m_{I}^{2}=q_{I}\left|\frac{F_{X}}{2 X_{R}}\right|^{2}, \quad A_{I J K}=\left(q_{I}+q_{J}+q_{K}\right) \frac{F_{X}}{2 X_{R}} .
$$

Here we used the fact that $\lambda_{I J K}$ is modulus independent due to the shift symmetry. It is noted that this tree-level expression derived at the cut-off scale $\Lambda$ of the SUGRA description does not have contribution from $F_{\Phi}$ because of the classical invariance of the Lagrangian under the superWeyl transformation. However, since the super-Weyl transformation is anomalous [2]-[4], we have $F_{\Phi}$ dependent terms at one-loop level via,

$$
f_{a}(X) \rightarrow f_{a}\left(\frac{\mu}{\Phi}, X\right), \quad\left(X+X^{\dagger}\right)^{q_{I}} \rightarrow\left(X+X^{\dagger}\right)^{q_{I}} Z_{I}\left(\frac{\mu}{|\Phi|}, X+X^{\dagger}\right),
$$

where $\mu$ represents the renormalization scale. This is the anomaly mediation [2] [3] which is loop suppressed and works whenever $F_{\Phi} \neq 0$ even without the modulus contribution. Including this effect, it is noticed that the gaugino mass reproduces the original tree-level value at, so-called, mirage messenger scale, $M_{\mathrm{mms}}=\Lambda\left(m_{3 / 2} / M_{P l}\right)^{\alpha / 2 c_{\lambda a}}$, which is independent of the choice of gauge group if $c_{\lambda_{a}}$ is universal [10]. This is a consequence of cancellation between the quantum correction to the modulus mediation and the anomaly mediation. The scalar mass parameters also show similar behavior at one-loop level if the relevant Yukawa couplings are negligible or $c_{\lambda_{a}}=1$, $q_{I}+q_{J}+q_{K}=1$ are satisfied. The name of the mirage mediation is taken from the fact that the scale, $M_{\mathrm{mms}}$, does not correspond to any physical threshold, although the soft parameters show the systematic pattern (eq.(11), (12)) at this scale, suggesting enhanced symmetry.

\section{The model}

Let us consider the hadronic axion model [25] in the above set-up by introducing the axion superfield $S$ and $N$ pairs of messenger fields $\Psi$ and $\bar{\Psi}, Q_{I}=\left(S, \Psi, \bar{\Psi}, Q_{i}\right)$, where we use a small 
subscript, $i$, to denote matter fields in MSSM. Here, $S$ is completely singlet under any unbroken gauge symmetry and $\Psi$ and $\bar{\Psi}$ are vectorlike representation of the SU(5) gauge group. We assigned the PQ charge as $Q_{\mathrm{PQ}}(S)=-2, Q_{\mathrm{PQ}}(\Psi)=Q_{\mathrm{PQ}}(\bar{\Psi})=1$ and $Q_{\mathrm{PQ}}(X)=0$. The superpotential has Yukawa coupling,

$$
W=\lambda \hat{S} \hat{\Psi} \hat{\bar{\Psi}}+\cdots
$$

which is allowed by the PQ symmetry. $S$ is a flat direction in SUSY limit, however, it is lifted by the SUSY breaking, $F_{X, \Phi}$, caused by the mirage mediation. We assume that $S$ is stabilized far away from the origin, breaking the PQ symmetry. This gives a huge mass for the messengers, so that they are integrated out at $\mu=|\langle\hat{S}\rangle| \simeq f_{\mathrm{PQ}}$, leaving $\hat{S}$ dependence in $f$ and $Z_{I}$ at low energy 39],

$$
f\left(\frac{\mu}{\Phi}, \frac{\hat{S}}{\Phi}, X\right), \quad Z_{i}\left(\frac{\mu}{|\Phi|}, \sqrt{\frac{\hat{S}^{\dagger} \hat{S}}{\Phi^{\dagger} \Phi}}, X+X^{\dagger}\right) .
$$

This introduces interactions among saxion/axino and the SM fields suppressed by $f_{\mathrm{PQ}}$, which is much stronger than those by $M_{\mathrm{Pl}}$. Later we will see these interactions play important roles in cosmology.

The scalar potential of $S$ is derived from the Kähler potential,

$$
\mathcal{L}=\int d^{4} \theta\left(X+X^{\dagger}\right)^{k} Z_{S}\left(\sqrt{\frac{\hat{S}^{\dagger} \hat{S}}{\Phi^{\dagger} \Phi}}, X+X^{\dagger}\right)|\hat{S}|^{2}
$$

where $Z_{S}$ is the wave function renormalization of $S$ at $\mu=|\hat{S}|$ and $k \equiv q_{S}$. We can obtain the relation among $F$-terms from the equation of motion of $F_{\hat{S}}$ as

$$
\frac{F_{\hat{S}}}{\hat{S}} \simeq-k \frac{F_{X}}{2 X_{R}}+\frac{1}{2} \frac{\partial \ln Z_{S}}{\partial \ln |\hat{S}|} F_{\Phi}
$$

Here and in what follows, we use an abbrebiation, $F^{\Phi} /\langle\Phi\rangle \rightarrow F_{\Phi}$ for the sake of brevity. It is noted that $F_{X} / 2 X_{R}$ is smaller than $F_{\Phi}$ by one-loop factor in the ballpark, while $\partial \ln Z_{S} / \partial \ln |\hat{S}|=$ $\gamma_{S} /\left(8 \pi^{2}\right)$ corresponds to the anomalous dimension of $S$ at $\mu=|\hat{S}|$ suppressed by the same factor. Integrating out the auxiliary component of $S$, the scalar potential is obtained as

$$
V(|\hat{S}|) \simeq\left\{k\left|\frac{F_{X}}{2 X_{R}}\right|^{2}-\frac{1}{4} \frac{\partial^{2} \ln Z_{S}}{\partial(\ln |\hat{S}|)^{2}}\left|F_{\Phi}\right|^{2}+\frac{1}{2}\left(\frac{\partial^{2} \ln Z_{S}}{\partial X \partial \ln |\hat{S}|} F_{\Phi}^{\dagger} F_{X}+\text { h.c. }\right)\right\}|\hat{S}|^{2}=m_{S}^{2}|\hat{S}|^{2}
$$

The extremum of this potential is determined by the condition,

$$
\frac{\partial V(|\hat{S}|)}{\partial|\hat{S}|}=\left[\left(2+\frac{\partial}{\partial \ln |\hat{S}|}\right) m_{S}^{2}\right]|\hat{S}|=0
$$


If $m_{S}^{2}(\mu)$ crosses zero at some scale $\mu=\mu_{0}$, we expect this condition holds in the vicinity of $|\hat{S}|=\mu_{0}$ since $\partial m_{S}^{2} / \partial \ln |\hat{S}| \sim m_{S}^{2} / 8 \pi^{2} \ll m_{S}^{2}$. In the case of the deflected anomaly mediation [39] which does not include the modulus contribution we have introduced, the $S$ potential is given by the second term in the parenthesis of eq.(18) [30],

$$
-\frac{1}{4} \frac{\partial^{2} \ln Z_{S}}{\partial(\ln |\hat{S}|)^{2}}\left|F_{\Phi}\right|^{2} \simeq-\left(\frac{1}{16 \pi^{2}}\right)^{2} N\left[16 g_{3}^{2}(S)-5(5 N+2) \lambda^{2}(S)\right] \lambda^{2}(S)\left|F_{\Phi}\right|^{2}
$$

where we have neglected the gauge coupling constants except the strong coupling, $g_{3}$, and $\lambda$ is normalized canonically as $\lambda^{2}(S)=\lambda^{2} /\left(X+X^{\dagger}\right)^{k+q_{\Psi}+q_{\bar{\Psi}}} Z_{S} Z_{\Psi} Z_{\bar{\Psi}}$. It is known that this form does not change by the RG evolution ${ }^{4}$. At a large value of $S$, the $\lambda^{4}$ term dominates and it leads to $m_{S}^{2}>0$, while at a small value, $m_{S}^{2}$ seems to become negative because of the asymptotic freedom of $g_{3}$. Therefore at first sight $S$ is expected to be stabilized at some scale $\langle S\rangle$ between the cut-off and the electroweak scale. However, in the case of the deflected anomaly mediation, we needed more than 3 flavors of messengers in order to avoid the tachyonic slepton [39]. Since the introduction of many number of messengers spoils the property of the asymptotic freedom, such a stabilization mechanism will not work without introducing new gauge interactions other than the SM. On the other hand, in our case, it is enough to introduce only one or two messengers because the tachyonic slepton problem has already been solved by the modulus mediation contribution. Furthermore, the positive contributions to the messenger masses, $m_{\Psi, \bar{\Psi}}^{2}$ and $A$-term, $A_{S \Psi \bar{\Psi}}$ from the modulus mediation (eq.(12)) drive $m_{S}^{2}$ to negative more strongly than those of the deflected anomaly mediation as read from the RG equation,

$$
\frac{d m_{S}^{2}}{d \ln \mu}=\frac{5 N \lambda^{2}}{8 \pi^{2}}\left(m_{\Psi}^{2}+m_{\bar{\Psi}}^{2}+m_{S}^{2}+\left|A_{S \Psi \bar{\Psi}}\right|^{2}\right) .
$$

Thus, the potential eq.(18) may have a minimum at a scale $\langle S\rangle$. Actually, if $c_{\lambda_{a}}=1, k=0$ and $q_{\Psi}+q_{\bar{\Psi}}=1$ are satisfied, $m_{S}^{2}$ crosses zero at $M_{\mathrm{mms}}$ irrelevant to the other parameters in the model as discussed in the previous section [10]. According to the RG equation this is at least a local minimum as far as $m_{\Psi}^{2}+m_{\bar{\Psi}}^{2} \gtrsim 0$, which is likely satisfied with the enhancement by the strong interaction. If we increase (or decrease) $q_{\Psi}+q_{\bar{\Psi}}$ breaking the mirage condition, the minimum will sit above (or below) $M_{\mathrm{mms}}$. In fig.1 we show the $S$ potential for the gauge kinetic function, $f \propto X\left(c_{\lambda_{a}}=1\right)$ with $N=1, k=0, q_{\Psi}=q_{\bar{\Psi}} \equiv \ell=1 / 2$ and $\alpha=1$. The minimum comes slightly below $M_{\mathrm{mms}}$ due to the one loop correction in the derivative of the $S$ potential.

The PQ scale $f_{\mathrm{PQ}} \simeq\langle S\rangle$ is constrained by various astrophysical and cosmological considerations. The relic abundance of the axion is given as [52]

$$
\Omega_{a} h^{2} \simeq 0.7\left(\frac{f_{\mathrm{PQ}}}{10^{12} \mathrm{GeV}}\right)^{7 / 6}\left(\frac{\Theta}{\pi}\right)^{2}
$$

\footnotetext{
${ }^{4}$ Precise treatment requires a separation of the triplet and doublet components in $\Psi / \hat{\Psi}$ and $\lambda$. We take this into account in the following numerical calculation.
} 
where $\Theta$ is the misalignment angle and $h$ the Hubble constant in unit of $100 \mathrm{Km} / \mathrm{sec} / \mathrm{Mpc}$. Apart from our ignorance of $\Theta$, to avoid the axion overclosure, $f_{\mathrm{PQ}} \lesssim 10^{12-13} \mathrm{GeV}$. On the other hand, the cooling of the SN 1987A puts a lower bound, $f_{\mathrm{PQ}} \gtrsim 10^{9} \mathrm{GeV}$ [53]. From these observations, $f_{\mathrm{PQ}}$ should be in the range of $10^{9} \mathrm{GeV} \lesssim f_{\mathrm{PQ}} \lesssim 10^{12-13} \mathrm{GeV}$. Let us examine whether this bound can be satisfied by our model or not. In fig.2, we depict $\langle S\rangle$ as a function of $\alpha$ for $c_{\lambda_{a}}=1, N=1$, $k=0$ and three rational choices of $\ell=0,1 / 2,1$. In the KKLT set-up with the uplifting by the anti-D3 brane $(\alpha \simeq 1)$, the SN bound on $f_{\mathrm{PQ}}$ is marginally satisfied with $\ell=1 / 2$ because $m_{S}^{2}$ crosses zero at $M_{\mathrm{mms}} \simeq 10^{10} \mathrm{GeV}$ for $\alpha \simeq 1$. While stepping up to $\ell=1$ the observed DM could be saturated by axion taking into account involved ambiguities. It is plausible that $\ell$ sits in the range $0<\ell<1$ in the string effective theory because the real part of the modulus represents the volume of the extra-dimension where the gauge fields propagate and $\ell$ is the scaling dimension against this volume for the matter kinetic term which couples to these gauge fields. Combining this with the axion window we obtain a constraint, $0.3 \lesssim \alpha \lesssim 1.5$ in this set-up. We numerically checked that these results are robust against changes in $N$ and $\lambda$ because both $m_{S}^{2}(\Lambda)$ and its RG equation depend them mostly through the overall factor $N \lambda^{2}$. Once we turn on $k>0$, the modulus contribution to $m_{S}^{2}(\Lambda)$ becomes competitive and defers its crossing zero in RG evolution, leading to lower $f_{\mathrm{PQ}}$. Thus $k=0$ is only allowed choice for $\alpha \simeq 1$ unless $\lambda$ is sufficently large ( $\left.\gtrsim 1\right)$ to dilute the modulus contribution. To push up $f_{\mathrm{PQ}}$ for $k>0$ or $\alpha \gtrsim 1.5$ (e.g. [41] [54]-[56]), we may need to introduce new gauge interactions which couples to the messengers and enhance $m_{\Psi / \bar{\Psi}}^{2}$ significantly via $R G$ evolution.

Next we estimate the physical masses of the component fields in $\hat{S}$. The scalar component is decomposed into the saxion $\sigma$ and the axion $a$, so that $\hat{S}=f_{\mathrm{PQ}} \exp \left[(\sigma+i a) / \sqrt{2} f_{\mathrm{PQ}}\right]$. The mass of the saxion $\sigma$ at the minimum of the potential is given by,

$$
\begin{aligned}
m_{\sigma}^{2} & =\left.\frac{1}{2} \frac{\partial^{2} V(|\hat{S}|)}{\partial|\hat{S}|^{2}}\right|_{\hat{S}=\langle\hat{S}\rangle}=\frac{1}{2}\left(1+\frac{\partial}{\partial \ln |\hat{S}|}\right)\left(2+\frac{\partial}{\partial \ln |\hat{S}|}\right) m_{S}^{2} \\
& \simeq \frac{\partial m_{S}^{2}}{\partial \ln |\hat{S}|} \simeq \frac{5 N \lambda^{2}}{8 \pi^{2}}\left(m_{\Psi}^{2}+m_{\bar{\Psi}}^{2}+\left|A_{\lambda}\right|^{2}\right)
\end{aligned}
$$

where we used eq.(19) to derive the second line. Assuming that $m_{\Psi}^{2}$ and $m_{\bar{\Psi}}^{2}$ are in the same order of magnitude with other superparticles, $m_{\sigma}^{2}$ is one-loop suppressed against the other soft breaking mass squared. From eq.(16), the mass of the axino $\widetilde{a}$, which is the superpartner of the 
axion, is calculated by

$$
\begin{aligned}
\mathcal{L} & =\int d^{4} \theta\left(X+X^{\dagger}\right)^{k} Z_{S}\left(\sqrt{\frac{\hat{S}^{\dagger} \hat{S}}{\Phi^{\dagger} \Phi}}, X+X^{\dagger}\right)|\hat{S}|^{2} \\
& =\int d^{4} \theta\left(X+X^{\dagger}\right)^{k}\left\{Z_{S}+\frac{1}{2} \frac{\partial Z_{S}}{\partial \ln |\hat{S}|} \ln \frac{\hat{S}^{\dagger} \hat{S}}{\Phi^{\dagger} \Phi}+\frac{1}{8} \frac{\partial^{2} Z_{S}}{\partial(\ln |\hat{S}|)^{2}}\left(\ln \frac{\hat{S}^{\dagger} \hat{S}}{\Phi^{\dagger} \Phi}\right)^{2}+\left(\frac{\partial Z_{S}}{\partial X} F_{X} \theta^{2}+\text { h.c. }\right)\right\}|\hat{S}|^{2} \\
& \simeq\left[\frac{1}{2} \frac{\partial^{2} \ln Z_{S}}{\partial \ln |\hat{S}| \partial X^{\dagger}} F_{X}^{\dagger}\left\langle\frac{\hat{S}^{\dagger}}{\hat{S}}\right\rangle-\frac{1}{4} \frac{\partial^{2} \ln Z_{S}}{\partial(\ln |\hat{S}|)^{2}} F_{\Phi}^{\dagger}\left\langle\frac{\hat{S}^{\dagger}}{\hat{S}}\right\rangle\right] \widetilde{a} \widetilde{a}
\end{aligned}
$$

where in the last line we have normalized the fields canonically. Thus, the axino mass is

$$
\begin{aligned}
m_{\widetilde{a}} & =\frac{1}{8 \pi^{2}}\left(\frac{1}{2} \frac{\partial \gamma_{S}(|\hat{S}|)}{\partial X^{\dagger}} F_{X}^{\dagger}-\frac{1}{4} \dot{\gamma_{S}}(|\hat{S}|) F_{\Phi}^{\dagger}\right)\left\langle\frac{\hat{S}^{\dagger}}{\hat{S}}\right\rangle \\
& =-\frac{5 N}{16 \pi^{2}} \lambda^{2}(S) A_{S \Psi \bar{\Psi}}(S)\left\langle\frac{\hat{S}^{\dagger}}{\hat{S}}\right\rangle
\end{aligned}
$$

where $\dot{\gamma}_{S} \equiv\left(d \gamma_{S} / d \ln \mu\right)$ and $A_{S \Psi \bar{\Psi}}$ is the sum of the $A$ terms of the mirage and the anomaly mediation. One can find that the axino mass arises at two-loop order in contrast with the masses of the other fields being at one-loop. Thus it will be LSP and candidate for DM. We discuss later how heavy is the axino which can explain the present DM abundance.

\section{The $\mu$-/B $\mu$-problem}

An important property of this model is that it provides a natural solution to the $\mu$-/ $B \mu$-problem (Here, we used the same notation $\mu$ for both the higgsino mass and the renormalization scale). To obtain the phenomenologically viable model, the $\mu$-term and the $B \mu$-term should be generated at the soft mass order. Let us consider the following superpotential and $\Omega$ function (See, for instance, [30] [39])

$$
\begin{gathered}
W=y_{1} T H_{1} H_{2}+y_{2} S_{1} S_{2} T \\
\Omega=\left|S_{1}\right|^{2}+\left|S_{2}\right|^{2}+|T|^{2}+\kappa S_{1}^{\dagger} S_{2}+\text { h.c. }
\end{gathered}
$$

where we introduced new singlets $S_{1}, S_{2}$ and $T$, whose PQ charge is assigned as $Q_{\mathrm{PQ}}\left(S_{1}\right)=$ $Q_{\mathrm{PQ}}\left(S_{2}\right)=-2$ and $Q_{\mathrm{PQ}}(T)=+4$, and $y_{1}, y_{2}$ and $\kappa$ are constants ${ }^{5}$. For simplicity, the modular weight of singlets is set to be zero. The assignment of PQ charge to new singlets leads to $Q_{\mathrm{PQ}}\left(H_{1}\right)=Q_{\mathrm{PQ}}\left(H_{2}\right)=-2$. This implies that we cannot obtain the $\mu$-term from the following Kähler potential: $\Delta K=\xi\left(X+X^{\dagger}\right)^{-n} H_{1} H_{2}$ with constant $\xi$ [57]. The last two terms in eq.(27)

\footnotetext{
${ }^{5}$ Here, one can interpret the new singlet $S_{1}$ or $S_{2}$ as the previous one $S$.
} 
are, in general, cannot be forbidden by the PQ symmetry ${ }^{6}$. As was discussed in the previous section, the Yukawa coupling of the messengers with $S_{1}$ yields a non-vanishing VEV for $S_{1}$. Then, $S_{2}$ and $T$ become massive and we can integrate out $T$. Its equation of motion leads to

$$
S_{2} \simeq-\frac{y_{1}}{y_{2}} \frac{H_{1} H_{2}}{S_{1}}
$$

Substituting eq.(28) into eq.(27), we can obtain

$$
\mathcal{L}=-\kappa \frac{y_{1}}{y_{2}} \int d^{4} \theta \frac{\hat{S}_{1}^{\dagger}}{\hat{S}_{1}} H_{1} H_{2}+\text { h.c. }
$$

Thus, the operator eq.(29) generates the $\mu$-/B $\mu$-term in canonical normaization:

$$
\begin{gathered}
\mu=-\frac{\kappa}{\left(X+X^{\dagger}\right)^{\left(q_{H_{1}}+q_{H_{2}}\right) / 2}} \frac{y_{1}}{y_{2}} \frac{F_{\hat{S}_{1}}^{\dagger}}{\hat{S}_{1}} \\
B \mu=\frac{\kappa}{\left(X+X^{\dagger}\right)^{\left(q_{H_{1}}+q_{H_{2}}\right) / 2}} \frac{y_{1}}{y_{2}} \frac{F_{\hat{S}_{1}}^{\dagger}}{\hat{S}_{1}}\left[\frac{F_{\hat{S}_{1}}}{\hat{S}_{1}}+\left(q_{H_{1}}+q_{H_{2}}\right) \frac{F_{X}}{2 X_{R}}\right],
\end{gathered}
$$

where we neglected field dependence in $\kappa$ and sub-leading terms from $Z_{H_{1}, H_{2}}{ }^{7}$. Since they are the same order of soft masses, the $\mu$ - $/ B \mu$-problem can be solved. It is noted that in our model there is also no SUSY CP problem. Since the phases of two SUSY-breaking $F$-terms, $F_{\Phi}$ and $F_{X}$, can be aligned [8] [9] [45, we find that the phase of $B$, which is obtained as

$$
B=-\frac{F_{\hat{S}_{1}}}{\hat{S}_{1}}-\left(q_{H_{1}}+q_{H_{2}}\right) \frac{F_{X}}{2 X_{R}} \simeq\left(k-q_{H_{1}}-q_{H_{2}}\right) \frac{F_{X}}{2 X_{R}}-\frac{1}{2} \frac{\partial \ln Z_{S}}{\partial \ln |\hat{S}|} F_{\Phi},
$$

can be rotated away simultaneously with that of the gaugino mass and $A$-term. Therefore, we constructed a model in which there are no $\mu$-problem as well as SUSY CP problem, and it leads to the axino LSP which is lighter than the neutralino. The appearance of the lighter LSP than the neutralino is favorable for the cosmological point of view. As we mentioned earlier, the moduli problem may be solved if there is the LSP which is lighter than the neutralino.

So far, we have assumed that the singlet is stabilized by messengers as explained in the previous section. However, now the extra singlets can play the role of messengers, and the light quarks have PQ charges [26]. Thus, with sufficiently large Yukawa couplings, we can also construct a model without messengers.

\footnotetext{
${ }^{6}$ When we write down in the superpotential all terms that are allowed by the PQ symmetry with minimal Kähler potential, we find that those terms can be translated into the form of eq.(26) and eq.(27). We also find that the coefficient $\kappa$ does not necessarily become small when $y_{1}$ and $y_{2}$ come to be small.

${ }^{7}$ This corresponds to the radiative correction to $\mu$ and $B \mu$. Note that if $y_{1,2}$ is sufficiently small the above results correctly give the input of (virtual) RG running at the unification scale in MSSM, although these terms are actually generated at $\mu=\left\langle S_{1}\right\rangle$.
} 


\section{Cosmology}

In this section, we discuss the cosmological implications of our model, in particular we shall closely investigate how it solves the LSP over-abundance problem due to gravitino decays. Since contributions to SUSY breaking from $S$ and $X$ are suppressed, we need to introduce an additional source of SUSY breaking, in order to cancel the cosmological constant. In KKLT, SUSY is broken by an anti- $D 3$ brane. Otherwise an additional field $Z$ is introduced. If supersymmetry breaking is of dynamical origin, its mass will be much higher than the electroweak scale. In what follows, we assume this is the case. During the inflationary epoch, the scalar fields such as $Z, X$ and $\sigma$ are deviated from their true minimum with some magnitudes. After inflation, each of fields will start a coherent oscillation when the Hubble parameter, $H$, becomes comparable to its mass except for the saxion. The behavior of the saxion is exceptional, which we will discuss shortly. The oscillation energy behaves like a matter, whose energy density is red shifted more slowly than that of radiation, so that it may dominate the energy of the Universe if the initial amplitude is not so small compared with the Planck scale. It is plausible that the modulus has the initial amplitude of the order of the Planck scale, while that of $Z$ is not the order of the Planck scale but the intermediate scale much smaller. Through out this paper, we assume that $Z$ decays sufficiently fast not to dominate the Universe. The coherent oscillation of the modulus would commence before the reheating due to the inflaton, $\phi_{I}$, decay is completed, if the reheating temperature is not so high. On the other hand, the time when the saxion starts to oscillate depends on whether the saxion is trapped at the origin or deviates from the origin during the inflation.

First, we consider the case where the saxion field is trapped at the origin during the inflation. This happens if the Hubble induced mass squared for the saxion at the inflationary era is positive. After the inflation, the Universe is reheated. The thermal effect through the messenger fields generates the temperature dependent effective potential for the saxion field. The resulting positive mass squared proportional to the temperature squared confines it at the origin until the temperature $T$ goes down to $T=T_{C} \sim 1 \mathrm{TeV}$ where the thermal effect ceases to dominate over the zero-temperature contribution eq.(23). The ratio of the energy density of the saxion to the entropy density at this time is

$$
\begin{aligned}
\frac{\rho_{\sigma}}{s} & \simeq \frac{45}{4 \pi^{2}} \frac{m_{\sigma}^{2}\langle\hat{S}\rangle^{2}}{g_{*} T_{C}^{3}} \\
& \simeq 1.1 \times 10^{13} \mathrm{GeV}\left(\frac{g_{*}\left(T_{C}\right)}{100}\right)^{-1}\left(\frac{T_{C}}{1 \mathrm{TeV}}\right)^{-3}\left(\frac{m_{\sigma}}{100 \mathrm{GeV}}\right)^{2}\left(\frac{\langle\hat{S}\rangle}{10^{10} \mathrm{GeV}}\right)^{2}
\end{aligned}
$$

On the other hand, since the modulus commences the coherent oscillation at the time when $H \simeq m_{X}$,

$$
\begin{aligned}
\frac{\rho_{X}}{s} & \simeq \frac{45}{4 \pi^{2}} \frac{m_{X}^{2} M_{\mathrm{Pl}}^{2}}{g_{*}\left(m_{X} M_{\mathrm{Pl}}\right)^{3 / 2}} \\
& \simeq 1.7 \times 10^{10} \mathrm{GeV}\left(\frac{g_{*}}{100}\right)^{-1}\left(\frac{m_{X}}{10^{6} \mathrm{GeV}}\right)^{1 / 2}
\end{aligned}
$$


The ratios remain constant until these fields decay unless other entropy productions occur. Comparing eq.(33) with eq.(34), we find the energy density of the saxion is much larger than that of the modulus, and hence the saxion dominates the Universe. The saxion mainly decays into a pair of axions with the decay width

$$
\Gamma_{\sigma} \simeq \Gamma(\sigma \rightarrow a a)=\frac{1}{64 \pi} \frac{m_{\sigma}^{3}}{\langle\hat{S}\rangle^{2}} .
$$

Since the axions produced by the saxion behave like neutrinos, they increase the Hubble expansion rate, and hence, the abundance of ${ }^{4} \mathrm{He}$. Thus, once the saxion dominates the Universe it would upset BBN unless there is an extra entropy production (e.g. thermal inflation [58]) after its decay.

Next, we consider the case where the saxion is displaced from the origin during the inflation. In this case, the saxion has at most the initial amplitude of the order of $M_{\mathrm{Pl}}, S_{\mathrm{in}} \lesssim M_{\mathrm{Pl}}$, and will start to oscillate when $H \simeq m_{\sigma}$. We should note that the thermal effect generates the effective potential with positive curvature around the origin. The effect becomes irrelevant for the field value of saxion larger than the temperature because the messenger fields acquires masses larger than the temperature and the contribution to the effective potential is suppressed. How the saxion zero-mode moves under this thermal potential would be complicated, investigation of which is beyond the scope of this paper. Being aware of the possibility that the saxion field may be trapped at the origin, we assume that this is not the case and the saxion field follows simple damped-coherent oscillation with the initial amplitude of the order of the Planck scale. After the reheating of $\phi_{I}$, since the oscillation energies of $X$ and $\sigma$ soon dominate the energy density of the Universe because of its relatively long lifetime. Their energy densities after the reheating are

$$
\frac{\rho_{X}\left(t_{d}^{I}\right)}{s} \simeq \frac{1}{8} T_{R}^{I} X_{\mathrm{in}}^{2}, \quad \frac{\rho_{\sigma}\left(t_{d}^{I}\right)}{s} \simeq \frac{1}{8} T_{R}^{I} S_{\mathrm{in}}^{2}
$$

where $t_{d}^{I}$ is the time when the inflaton decays and $T_{R}^{I}$ the reheating temperature of the inflation. Here we consider the case both $\mathrm{X}$ and $\sigma$ start coherent oscillation during the era of inflation coherent oscillation (before the reheating of the $\phi_{I}$ ). However, since the decay constant of the saxion is much smaller than the Planck scale, the saxion decays much faster than the modulus. In fact, the lifetime of the saxion and the modulus is (See, eq.(135) and eq.(52))

$$
\begin{gathered}
\tau_{\sigma} \simeq 1.3 \times 10^{-8} \mathrm{sec} .\left(\frac{m_{\sigma}}{100 \mathrm{GeV}}\right)^{-3}\left(\frac{\langle\hat{S}\rangle}{10^{10} \mathrm{GeV}}\right)^{2}, \\
\tau_{X} \simeq 6.3 \times 10^{-5} \mathrm{sec}\left(\frac{m_{X}}{10^{6} \mathrm{GeV}}\right)^{-3},
\end{gathered}
$$

where we have set $d_{g}=1$. Therefore, even if the saxion has initial amplitude of the order of the Planck scale, the saxion decays faster than the modulus, and hence the modulus dominates the Universe as long as the confinement of the saxion in the thermal potential does not occur. When the modulus dominates the Universe, its decay produces a large amount of entropy. Let us estimate how large entropy is released by the modulus decay. When we denote that $t_{\mathrm{osc}}^{X}$ the 
time at which the coherent oscillation of the modulus commences, the ratio of the energy density of $X$ and that of the radiation at the modulus decay time, $t_{d}^{X}$, is

$$
\frac{\rho_{X}\left(t_{d}^{X}\right)}{\rho_{R}\left(t_{d}^{X}\right)}=\frac{\rho_{X}\left(t_{d}^{I}\right)}{\rho_{R}\left(t_{d}^{I}\right)} \frac{R\left(t_{d}^{X}\right)}{R\left(t_{d}^{I}\right)}=\frac{\rho_{X}\left(t_{\mathrm{osc}}^{X}\right)}{\rho_{\phi_{I}}\left(t_{\mathrm{osc}}^{X}\right)}\left(\frac{T_{R}^{I}}{T_{d}^{X}}\right)^{4 / 3} \simeq \frac{1}{6} X_{\mathrm{in}}^{2}\left(\frac{T_{R}^{I}}{T_{d}^{X}}\right)^{4 / 3}
$$

where $T_{d}^{X}$ is the decay temperature of $X$ and $R$ the scale factor. Therefore, unless the initial amplitude of $X$ is not much smaller than the Planck scale the entropy increase factor $\Delta$ is

$$
\Delta \equiv \frac{s_{\text {after }}\left(t_{d}^{X}\right)}{s_{\text {before }}\left(t_{d}^{X}\right)}=\left(\frac{\rho_{X}\left(t_{d}^{X}\right)}{\rho_{R}\left(t_{d}^{X}\right)}\right)^{3 / 4} \simeq X_{\text {in }}^{3 / 2} \frac{T_{R}^{I}}{T_{d}^{X}} \sim 10^{7},
$$

when, for instance, $T_{R}^{I} \simeq 10^{6} \mathrm{GeV}$ and $T_{d}^{X} \simeq 0.1 \mathrm{GeV}^{8}$. This relatively large entropy increase can sufficiently erase unwanted particles produced before the modulus decay. The axions produced by the saxion decay are also diluted. The ratio of the energy density of the axion, $\rho_{a}$, to that of the radiation at the modulus decay can be estimated as

$$
\frac{\rho_{a}\left(t_{d}^{X}\right)}{\rho_{R}\left(t_{d}^{X}\right)}=\frac{\rho_{a}\left(t_{d}^{\sigma}\right)}{\rho_{X}\left(t_{d}^{\sigma}\right)} \frac{R\left(t_{d}^{\sigma}\right)}{R\left(t_{d}^{X}\right)} \simeq\left(\frac{\tau_{\sigma}}{\tau_{X}}\right)^{2 / 3} \simeq 3 \times 10^{-3},
$$

where $t_{d}^{\sigma}$ is the decay time of the saxion and we have used eq.(37) and eq.(38) in the last step. In order that the produced axions do not change the Hubble expansion rate, its energy density should be less than that of the one neutrino species:

$$
\left.\frac{\rho_{a}}{\rho_{R}}\right|_{1 \mathrm{MeV}} \lesssim \frac{7}{43}
$$

Comparing eq.(41) and eq.(42), the axions produced by the saxion would not spoil BBN. Therefore, we find that it is sufficient to discuss the cosmic evolution only after the modulus decay in the case where both the modulus and the saxion have the initial amplitude of the order of the Planck scale. In what follows, we address the implications of the modulus decay to cosmology.

The modulus mainly decays into gauge multiplets through the gauge kinetic function. (See, the second term in the last line in eq.([6).) The decay width into gauge boson pairs and into gaugino pairs is [17] [18]

$$
\Gamma(X \rightarrow g g)=\Gamma(X \rightarrow \lambda \lambda)=\frac{3}{32 \pi}\left(\frac{N_{G}}{12}\right) d_{g}^{2} \frac{m_{X}^{3}}{M_{\mathrm{Pl}}^{2}},
$$

where $N_{G}$ is the number of the gauge bosons, which is 12 for MSSM. The numerical factor of order unity, $d_{g}$, is defined by

$$
d_{g} \equiv\left\langle G_{X X^{\dagger}}\right\rangle^{-\frac{1}{2}}\left\langle f_{R}\right\rangle^{-1}\left|\left\langle\frac{\partial f}{\partial X}\right\rangle\right|
$$

\footnotetext{
${ }^{8}$ The modulus decay temperature with $m_{X} \simeq 10^{6} \mathrm{GeV}$ is of the order of $0.1 \mathrm{GeV}$. See below.
} 
where the subscript $X$ represents derivative with respect to $X$ and the real part $f_{R}=\operatorname{Re} f$, respectively. The function $G$ is the total Kähler potential defined by $G \equiv K+\ln |W|^{2}$. Remarkably it was recently recognized that the decay of $X$ into the gravitino pair is not suppressed. The decay width is given by [17] [18 ${ }^{9}$

$$
\Gamma\left(X \rightarrow \psi_{3 / 2} \psi_{3 / 2}\right)=\frac{d_{3 / 2}^{2}}{288 \pi} \frac{m_{X}^{3}}{M_{\mathrm{Pl}}^{2}},
$$

where $d_{3 / 2}$ is also order one coefficient defined by

$$
\left\langle G_{X X^{\dagger}}\right\rangle^{-1 / 2}\left\langle e^{G / 2} G_{X}\right\rangle \equiv d_{3 / 2} \frac{m_{3 / 2}^{2}}{m_{X}} .
$$

The modulus also decays into the saxion pair and the axino pair. The relevant interaction for the decay into the saxion pair is

$$
\mathcal{L}_{X \sigma \sigma}=-\frac{1}{2}\left[\frac{k}{\left(2 X_{R}\right)^{2}} F_{X} \frac{\partial F_{X}^{\dagger}}{\partial X}+\frac{1}{2} \frac{\partial^{2} \ln Z_{S}}{\partial X^{\dagger} \partial \ln |\hat{S}|} F_{\Phi} \frac{\partial F_{X}^{\dagger}}{\partial X}\right] \delta X \sigma \sigma
$$

where $\delta X \equiv X-\langle X\rangle$. In eq.(47), the first term gives the dominant contribution because $\left\langle\partial F_{X}^{\dagger} / \partial X\right\rangle \simeq m_{X}$ and $\left\langle F_{X}\right\rangle \simeq m_{3 / 2}^{2} / m_{X}$. The decay width can be estimated as

$$
\Gamma(X \rightarrow \sigma \sigma) \simeq \frac{k^{2}}{128 \pi}\left(\frac{m_{3 / 2}}{m_{X}}\right)^{4} \frac{m_{X}^{3}}{M_{\mathrm{Pl}}^{2}}
$$

The interaction of $X$ with axino comes from eq.(24)

$$
\mathcal{L}_{X \widetilde{a} \widetilde{a}} \simeq \frac{1}{2} \frac{\partial^{2} \ln Z_{S}}{\partial \ln |\hat{S}| \partial X^{\dagger}} \frac{\partial F_{X}^{\dagger}}{\partial X} \delta X \widetilde{a} \widetilde{a}
$$

Since the derivative of the anomalous dimension with respect to $X$ is

$$
\frac{1}{2} \frac{\partial^{2} \ln Z_{S}}{\partial \ln |\hat{S}| \partial X^{\dagger}} \simeq \frac{5 N \lambda^{2}}{16 \pi^{2}}
$$

the decay width into the axino pair is

$$
\Gamma(X \rightarrow \widetilde{a} \widetilde{a}) \simeq \frac{1}{128 \pi}\left(\frac{5 N \lambda^{2}}{16 \pi^{2}}\right)^{2} \frac{m_{X}^{3}}{M_{\mathrm{Pl}}^{2}} .
$$

\footnotetext{
${ }^{9}$ In some cases where $Z$ is light and has a minimal coupling, the mixing between $Z$ and $X$ might play an important role [59] 60, suppressing the decay of $X$ into the gravitinos. However since $m_{Z} \gg m_{X}$, this does not work in our case.
} 
Comparing these partial decay widths, we find that the modulus dominantly decays into gauge boson/gaugino pairs with the total decay width

$$
\Gamma_{X} \simeq \frac{3}{16 \pi}\left(\frac{N_{G}}{12}\right) d_{g}^{2} \frac{m_{X}^{3}}{M_{\mathrm{Pl}}^{2}} .
$$

Thus, the reheating temperature of $X$ is

$$
\begin{aligned}
T_{R}^{X} & =\left(\frac{90}{\pi^{2} g_{*}\left(T_{R}^{X}\right)}\right)^{1 / 4} \sqrt{\Gamma_{X} M_{\mathrm{Pl}}} \\
& =1.5 \times 10^{2} \mathrm{MeV}\left(\frac{g_{*}\left(T_{R}^{X}\right)}{10}\right)^{-1 / 4}\left(\frac{N_{G}}{12}\right)^{1 / 2} d_{g}\left(\frac{m_{X}}{10^{6} \mathrm{GeV}}\right)^{3 / 2}
\end{aligned}
$$

where $g_{*}\left(T_{R}^{X}\right)$ is the effective degrees of freedom of the radiation at the reheating. Before discussing how the present DM abundance is composed, we should comment on saxions produced by the modulus decay. The branching ratio of $X$ into the saxion pair is obtained from eq.(48) and eq.(52)

$$
B_{\sigma}^{X} \equiv B r(X \rightarrow \sigma \sigma)=\frac{k^{2}}{24 d_{g}^{2}}\left(\frac{m_{3 / 2}}{m_{X}}\right)^{4} .
$$

The yield of the saxion produced by the modulus decay is

$$
\begin{aligned}
Y_{\sigma}^{X} & =\frac{3}{2} \frac{T_{R}^{X}}{m_{X}} B_{\sigma}^{X} \\
& \simeq 9.6 \times 10^{-13} \frac{k^{2}}{d_{g}}\left(\frac{g_{*}\left(T_{R}^{X}\right)}{10}\right)^{-1 / 4}\left(\frac{N_{G}}{12}\right)^{-1 / 2}\left(\frac{m_{X}}{10^{6} \mathrm{GeV}}\right)^{-7 / 2}\left(\frac{m_{3 / 2}}{10^{5} \mathrm{GeV}}\right)^{4} .
\end{aligned}
$$

However, from eq.(37), we find that the saxions decay immediately when they are produced by the modulus, and hence their decay does not spoil the success of BBN.

In our model, as we have already mentioned, the axino is the LSP and the DM candidate. Thus, in the following discussions, let us estimate the axino abundance and discuss whether the axino can explain the present DM abundance. There are four production mechanisms of axinos. We sketched these four processes in fig 3 . One of the processes is the production from the decay of the modulus directly. The second comes from the decay of next LSPs (NLSP), $\tilde{\chi}$, produced by the modulus. We should note that the sparticles are not in the thermal equilibrium because the reheat temperature after the $\mathrm{X}$ decay is not high enough. The abundance of axino produced by this process is essentially the same as that of NLSP, and hence we estimate the abundance of NLSP at the modulus decay. In the third process, axinos are produced by the decay of gravitinos. Since gravitinos decay uniformly into all MSSM particles and the axino, the axino abundance may be determined by the ratio of the decay channels. The fourth and last process is that NLSPs produced by gravitinos decay into axinos. We discuss these processes for cases where the NLSP is the bino, the higgsino and the stau in turn. 


\subsection{Bino NLSP case}

1st. process. The branching ratio of $X$ into the axino pair can be estimated from eq. (51) and eq.(152) as

$$
B_{\widetilde{a}}^{X} \equiv B r(X \rightarrow \widetilde{a} \widetilde{a}) \simeq \frac{1}{24 d_{g}^{2}}\left(\frac{5 N \lambda^{2}}{16 \pi^{2}}\right)^{2}
$$

According to eq.(56) and eq.(53), the relic abundance of axinos directly produced by the modulus decay is

$$
Y_{\widetilde{a}}^{X}=\frac{3}{2} \frac{T_{R}^{X}}{m_{X}} B_{\widetilde{a}}^{X} \simeq 9.3 \times 10^{-12} N^{2} \lambda^{4}\left(\frac{g_{*}\left(T_{R}^{X}\right)}{10}\right)^{-1 / 4} d_{g}^{-1}\left(\frac{N_{G}}{12}\right)^{1 / 2}\left(\frac{m_{X}}{10^{6} \mathrm{GeV}}\right)^{1 / 2} .
$$

For the axino mass below the electroweak scale, this yield will be too small to account for the present DM abundance.

2nd. process. When the modulus reheats the Universe, neutralinos are so abundant that the annihilation among them may become effective. However, since the neutralino can decay into the axino, if the decay width is larger than the interaction rate, then the annihilation process will not be effective. The bino decays into the axino and a photon or a $Z^{0}$-boson through the following coupling

$$
\mathcal{L}_{\tilde{\chi} \widetilde{a} \gamma}=\frac{\alpha_{1} N}{16 \sqrt{2} \pi} \frac{1}{\langle\hat{S}\rangle} \widetilde{\widetilde{a}} \gamma_{5}\left[\gamma^{\mu}, \gamma^{\nu}\right] \widetilde{B} B_{\mu \nu}
$$

where $N$ is the number of the messengers, $B_{\mu \nu}$ the field strength of the $\mathrm{U}(1)_{Y}$ gauge boson $B_{\mu}$. In this expression, we used the four component notation. Eq.(158) leads to [37]

$$
\begin{gathered}
\Gamma(\widetilde{B} \rightarrow \widetilde{a} \gamma) \simeq \frac{\alpha_{\mathrm{em}}^{2} N^{2}}{256 \pi^{3}} \frac{1}{\cos ^{2} \theta_{W}} \frac{m_{\widetilde{B}}^{3}}{\langle\hat{S}\rangle^{2}}, \\
\Gamma\left(\widetilde{B} \rightarrow \widetilde{a} Z^{0}\right) \simeq \frac{\alpha_{\mathrm{em}}^{2} N^{2}}{256 \pi^{3}} \frac{\tan ^{2} \theta_{W}}{\cos ^{2} \theta_{W}}\left(1-x_{Z}\right)\left(1-\frac{x_{Z}}{2}-\frac{x_{Z}^{2}}{2}\right) \frac{m_{\widetilde{B}}^{3}}{\langle\hat{S}\rangle^{2}},
\end{gathered}
$$

where $\alpha_{\mathrm{em}}$ is the fine structure constant, $\theta_{W}$ the Weinberg angle and $x_{Z} \equiv m_{Z^{0}}^{2} / m_{\tilde{\chi}}^{2}$ with $Z^{0}$-boson mass, $m_{Z^{0}}$, and the NLSP mass, $m_{\tilde{\chi}}$. When the bino is sufficiently heavy, its lifetime is relatively short

$$
\begin{aligned}
\tau_{\widetilde{B}} & =\Gamma_{\widetilde{B}}^{-1} \equiv\left(\Gamma(\widetilde{B} \rightarrow \widetilde{a} \gamma)+\Gamma\left(\widetilde{B} \rightarrow \widetilde{a} Z^{0}\right)\right)^{-1} \\
& \simeq 1.9 \times 10^{-4} \text { sec. }
\end{aligned}
$$

for $m_{\widetilde{B}}=300 \mathrm{GeV}$ and $\langle S\rangle=10^{10} \mathrm{GeV}^{10}$. Assuming that the bino is heavy enough to annihilate into a top quark pair through s-wave, the annihilation cross section of the bino, $\left\langle\sigma_{\text {ann. }} v_{\text {rel. }}\right\rangle_{\widetilde{B}}$, is

\footnotetext{
${ }^{10}$ In the mirage mediation, such a relatively heavy bino is usually realized.
} 
given by 61 .

$$
\left\langle\sigma_{\text {ann. }} v_{\text {rel. }}\right\rangle_{\widetilde{B}} \simeq \frac{32 \pi}{27} \alpha_{1}^{2} \frac{m_{t}^{2}}{\left(m_{\widetilde{t}_{R}}^{2}+m_{\widetilde{B}}^{2}-m_{t}^{2}\right)^{2}}\left(1-\frac{m_{t}^{2}}{m_{\widetilde{B}}^{2}}\right)^{1 / 2}
$$

with $m_{t}, m_{\widetilde{t}_{R}}$ and $m_{\widetilde{B}}$ being the masses of the top-quark, the right-handed stop and the bino, respectively. Here $\sigma_{\text {ann. }}$ is the annihilation cross section of two binos, $v_{\text {rel. }}$ their relative velocity and $\langle\cdots\rangle$ represents the thermal average ${ }^{11}$. Thus, from eq.(159) and eq.([61), we can find that the interaction rate at the modulus decay is much larger than the decay width:

$$
\left\langle\sigma_{\text {ann. }} v_{\text {rel. }}\right\rangle_{\widetilde{B}} n_{\widetilde{B}}=\left\langle\sigma_{\text {ann. }} v_{\text {rel. }}\right\rangle_{\widetilde{B}} Y_{\widetilde{B}} s \gg \Gamma_{\widetilde{B}} \text {, }
$$

where $n_{\widetilde{B}}$ is the number density of the bino. The annihilation process will terminate when the Hubble parameter becomes comparable to the annihilation rate

$$
\left\langle\sigma_{\text {ann. }} v_{\text {rel. }}\right\rangle_{\widetilde{B}} n_{\widetilde{B}} \simeq H\left(T_{R}^{X}\right) .
$$

The bino abundance after their annihilation is estimated as

$$
\left.\frac{n_{\widetilde{B}}}{s}\right|_{T_{R}^{X}} \simeq \frac{1}{4}\left(\frac{90}{\pi^{2} g_{*}}\right)^{1 / 2} \frac{1}{\left\langle\sigma_{\text {ann. }} v_{\text {rel. }}\right\rangle_{\widetilde{B}} T_{R}^{X} M_{\mathrm{Pl}}} .
$$

After the annihilation, the bino NLSP decays into the axino, and hence the axino abundance is the same as that of bino:

$$
\begin{aligned}
\left.Y_{\widetilde{a}}^{\widetilde{B}} \simeq \frac{n_{\widetilde{B}}}{s}\right|_{T_{R}^{X}} & \simeq 6.2 \times 10^{-19}\left(\frac{g_{*}\left(T_{R}^{X}\right)}{10}\right)^{-1 / 4} d_{g}^{-1}\left(\frac{N_{G}}{12}\right)^{-1 / 2}\left(\frac{m_{X}}{10^{6} \mathrm{GeV}}\right)^{-3 / 2} \frac{\mathrm{GeV}^{-2}}{\left\langle\sigma_{\text {ann. }} v_{\text {rel. }}\right\rangle_{\widetilde{B}}} \\
& \simeq 9.4 \times 10^{-10}\left(\frac{g_{*}\left(T_{R}^{X}\right)}{10}\right)^{-1 / 4} d_{g}^{-1}\left(\frac{N_{G}}{12}\right)^{-1 / 2}\left(\frac{m_{X}}{10^{6} \mathrm{GeV}}\right)^{-3 / 2},
\end{aligned}
$$

where we have set $m_{t}=174 \mathrm{GeV}, m_{\widetilde{B}}=300 \mathrm{GeV}$ and $m_{\widetilde{t}_{R}} / m_{\widetilde{B}}=1.25$ as the reference values.

3rd. process. A fraction of the gravitinos decays into axinos. Such an axino abundance is provided by the ratio of the decay channels. Since the total decay width of the gravitino with negligible final state mass is (see, for instance [62])

$$
\Gamma_{3 / 2}=\frac{244}{384 \pi} \frac{m_{3 / 2}^{3}}{M_{\mathrm{Pl}}^{2}}
$$

\footnotetext{
${ }^{11}$ If the bino is so light that it cannot annihilate into the top quark, since the annihilation cross section becomes proportional to their relative velocity, the cross section is more suppressed. In such a case, the relic abundance of the bino becomes more redundant, and hence in order to explain the present DM abundance, the axino mass should be smaller about one order or so than that in our case.
} 
the axino abundance can be estimated as

$$
\begin{aligned}
Y_{\widetilde{a}}^{3 / 2} & \simeq \frac{1}{244} Y_{3 / 2}^{X}=\frac{1}{244} \frac{3}{2} B_{3 / 2} \frac{T_{R}^{X}}{m_{X}} \\
& \simeq 1.7 \times 10^{-11}\left(\frac{g_{*}\left(T_{R}^{X}\right)}{10}\right)^{-1 / 4} d_{g}\left(\frac{N_{G}}{12}\right)^{1 / 2}\left(\frac{m_{X}}{10^{6} \mathrm{GeV}}\right)^{1 / 2}
\end{aligned}
$$

where $Y_{3 / 2}^{X}$ denotes the yield of the gravitino produced by the modulus decay. In the last step, we used the branching ratio into the gravitino pair $B_{3 / 2}$ which is defined by

$$
B_{3 / 2}=\frac{1}{54} \frac{d_{3 / 2}^{2}}{d_{g}^{2}}\left(\frac{N_{G}}{12}\right)^{-1} \simeq 0.01
$$

4th. process. Finally, let us consider the process that the binos produced by the gravitinos decay into axinos. In distinction to the case of the modulus decay, the decay temperature of the gravitino is not so high,

$$
\begin{aligned}
T_{3 / 2} & \simeq\left(\frac{90}{\pi^{2} g_{*}\left(T_{3 / 2}\right)}\right)^{1 / 4} \sqrt{\Gamma_{3 / 2} M_{\mathrm{Pl}}} \\
& \simeq 9.0 \times 10^{-3} \mathrm{GeV}\left(\frac{g_{*}\left(T_{3 / 2}\right)}{10}\right)^{-1 / 4}\left(\frac{m_{3 / 2}}{10^{5} \mathrm{GeV}}\right)^{3 / 2},
\end{aligned}
$$

and then, the annihilation process does not occur effectively. Therefore, the axino abundance is the same as that of the gravitinos:

$$
Y_{\widetilde{a}}^{\widetilde{B}} \simeq Y_{3 / 2}^{X} \simeq 4.3 \times 10^{-9}\left(\frac{g_{*}\left(T_{R}^{X}\right)}{10}\right)^{-1 / 4} d_{g}\left(\frac{N_{G}}{12}\right)^{1 / 2}\left(\frac{m_{X}}{10^{6} \mathrm{GeV}}\right)^{1 / 2}
$$

Comparing eqs.(57), (65), (67) and (70), we find that the axino abundance is dominantly constituted from the 4 th. process, that is, decay of the binos produced by the gravitinos, eq.(170).

We should estimate whether the binos can become non-relativistic by scattering with thermal bath [63] 64]. At the gravitino decay, binos lose its energy mainly by scattering with the background electron. When the bino is produced by the gravitino, it is relativistic, and then the cross section, $\sigma_{\text {scatt. }}$, is estimated by $[\underline{63}$

$$
\left\langle\sigma_{\text {scatt. }} v_{\text {rel. }}\right\rangle \simeq 128 \pi \alpha_{1}^{2} \frac{E_{\widetilde{B}}^{2} T_{3 / 2}^{2}}{m_{\widetilde{e}_{R}}^{4} m_{\widetilde{B}}^{2}},
$$

where $E_{\widetilde{B}}$ is the energy of the bino and $m_{\widetilde{e}_{R}}$ the mass of the right-handed selectron. The energy loss rate for the relativistic NLSP, $\Gamma_{\text {scatt. }}^{\mathrm{NLSP}}$, is given by

$$
\Gamma_{\text {scatt. }}^{\widetilde{B}} \simeq n_{e}\left\langle\sigma_{\text {scatt. }} v_{\text {rel. }}\right\rangle \frac{\Delta E_{\widetilde{B}}}{E_{\widetilde{B}}}
$$


where $n_{e}$ is the number density of the back-ground electron and $\Delta E_{\widetilde{B}} / E_{\widetilde{B}}$ the averaged energy loss rate of bino in one scattering, which is given by

$$
\frac{\Delta E_{\widetilde{B}}}{E_{\widetilde{B}}} \simeq 12\left(\frac{E_{\widetilde{B}} T_{3 / 2}}{m_{\widetilde{B}}^{2}}\right)
$$

Comparing the energy loss rate eq.(172) with the decay width eq.(159), we can find

$$
\left.\frac{\Gamma_{\text {scatt. }}^{\widetilde{B}}}{\Gamma_{\widetilde{B}}}\right|_{T_{3 / 2}} \sim 2 \times 10^{3}
$$

for $m_{\widetilde{B}} \simeq m_{\widetilde{e}_{R}}=300 \mathrm{GeV}, T_{3 / 2} \simeq 10 \mathrm{MeV}$ and $E_{\widetilde{B}} \simeq 50 \mathrm{TeV}$. Therefore, at the gravitino decay the binos decay into axinos after becoming non-relativistic without the annihilation process:

$$
\Gamma_{\text {scatt. }}^{\widetilde{B}} \gg \Gamma_{\widetilde{B}} \gg\left\langle\sigma_{\text {ann. }} v_{\text {rel. }}\right\rangle_{\widetilde{B}} n_{\widetilde{B}}
$$

\subsection{Higgsino NLSP case}

Let us next consider the case of the higgsino NLSP. The results of the 1st. and 3rd. processes remain valid, irrespective of the composition of NLSPs. A difference from the bino NLSP case is the annihilation cross section. It is given by 61 .

$$
\left\langle\sigma_{\text {ann. }} v_{\text {rel. }}\right\rangle_{\widetilde{h}} \simeq \frac{g_{2}^{4}}{32 \pi} \frac{1}{m_{\widetilde{h}}^{2}} \frac{\left(1-x_{W}\right)^{3 / 2}}{\left(2-x_{W}\right)^{2}}+\frac{g_{2}^{4}}{64 \pi \cos ^{4} \theta_{W}} \frac{1}{m_{\widetilde{h}}^{2}} \frac{\left(1-x_{Z}\right)^{3 / 2}}{\left(2-x_{Z}\right)^{2}}
$$

with $x_{W} \equiv m_{W}^{2} / m_{\tilde{\chi}}^{2}, m_{W}$ being the $W$-boson mass, $g_{2}$ the $\mathrm{SU}(2)$ gauge coupling constant and $m_{\widetilde{h}}$ the higgsino mass. Here coannihilation is not taken into account. The higgsino can decay into the axino and the Higgs boson through the $\mu$-term, eq.(29),

$$
\mathcal{L}=\kappa \frac{y_{1}}{y_{2}} \frac{1}{\langle\hat{S}\rangle}\left\{\left\langle\frac{F_{\hat{S}}^{\dagger}}{\hat{S}}\right\rangle\left(\widetilde{a} \widetilde{h}_{1}^{0} h_{2}^{0}+\widetilde{a} \widetilde{h}_{2}^{0} h_{1}^{0}\right)+\text { h.c. }\right\} .
$$

The physical neutral Higgs bosons are related to $h_{1}^{0}$ and $h_{2}^{0}$ by

$$
\begin{aligned}
& h_{1}^{0}=\frac{1}{\sqrt{2}}\left(v_{1}-h^{0} \sin \alpha+H^{0} \cos \alpha+i A^{0} \sin \beta+i G^{0} \cos \beta\right) \\
& h_{2}^{0}=\frac{1}{\sqrt{2}}\left(v_{2}+h^{0} \cos \alpha+H^{0} \sin \alpha+i A^{0} \cos \beta-i G^{0} \sin \beta\right)
\end{aligned}
$$

with $v_{1}$ and $v_{2}$ being VEVs of the $H_{1}$ and $H_{2}$. Here $h^{0}, H^{0}$ and $A^{0}$ are physical scalars, which correspond to the CP even light Higgs, the CP even heavy Higgs and the CP odd Higgs, respectively, and $G^{0}$ is the Nambu-Goldstone boson. The mixing angle $\alpha$ (which should not be confused with $\alpha$ parameter eq. (8) ) satisfies the relation

$$
\tan 2 \alpha=\frac{m_{A}^{2}+m_{Z^{0}}^{2}}{m_{A}^{2}-m_{Z^{0}}^{2}} \tan 2 \beta
$$


where $\tan \beta \equiv v_{2} / v_{1}$ and $m_{A}$ is the mass of $A^{0}$. Then, the decay width of the higgsino into the axino and the Higgs boson is

$$
\Gamma\left(\widetilde{h}_{ \pm} \rightarrow \widetilde{a} h^{0}\right) \simeq \frac{1}{32 \pi}(\cos \alpha \mp \sin \alpha)^{2}\left(1-\frac{m_{h^{0}}^{2}}{m_{\widetilde{h}}^{2}}\right)^{2}\left(\frac{\mu}{m_{\widetilde{h}}}\right)^{2} \frac{m_{\widetilde{h}}^{3}}{\langle S\rangle^{2}},
$$

where $\mu$ is the $\mu$-term, i.e., eq.(30) and $\widetilde{h}_{ \pm}$the mass eigenstate, which are defined as $\widetilde{h}_{ \pm} \equiv$ $\frac{1}{\sqrt{2}}\left(\widetilde{h}_{1}^{0} \pm \widetilde{h}_{2}^{0}\right)$. The higgsino can also decay into the axino and the $Z^{0}$ boson through the axinohiggsino mixing. According to eq.(29), the axino and the higgsino are mixed each other by

$$
\mathcal{L}_{\text {mix }}=-\kappa \frac{y_{1}}{y_{2}}\left\langle\frac{F_{\hat{S}}^{\dagger}}{\hat{S}^{2}}\right\rangle v_{2} \widetilde{a} \widetilde{h}_{1}^{0}-\kappa \frac{y_{1}}{y_{2}}\left\langle\frac{F_{\hat{S}}^{\dagger}}{\hat{S}^{2}}\right\rangle v_{1} \widetilde{a} \widetilde{h}_{2}^{0}+\text { h.c. }
$$

This mixing can be removed by transformations

$$
\begin{aligned}
\widetilde{a}^{\prime} & \simeq \widetilde{a}-\frac{\epsilon_{1}}{\mu} \widetilde{h}_{1}^{0}-\frac{\epsilon_{2}}{\mu} \widetilde{h}_{2}^{0}, \\
\widetilde{h}_{1}^{0 \prime} & \simeq \widetilde{h}_{1}^{0}+\frac{\epsilon_{1}}{\mu} \widetilde{a}, \\
\widetilde{h}_{2}^{0 \prime} & \simeq \widetilde{h}_{2}^{0}+\frac{\epsilon_{2}}{\mu} \widetilde{a},
\end{aligned}
$$

where we have neglected the higher power of $\epsilon_{i} / \mu \equiv-\kappa \frac{y_{1}}{y_{2}}\left\langle\frac{F_{\hat{S}}^{\dagger}}{\hat{S}^{2}}\right\rangle v_{i} / \mu \simeq v_{i} /\langle\hat{S}\rangle$. In the unitary gauge, the relevant term to the decay is

$$
\mathcal{L}=-\frac{g_{2}}{2 \cos \theta_{W}} \frac{\epsilon_{1}}{\mu} \overline{\widetilde{h}_{1}^{0}} \bar{\sigma}^{\mu} \widetilde{a} Z_{\mu}+\frac{g_{2}}{2 \cos \theta_{W}} \frac{\epsilon_{2}}{\mu} \overline{\widetilde{h}_{2}^{0}} \bar{\sigma}^{\mu} \widetilde{a} Z_{\mu}
$$

Thus, the decay width of the higgsino into the axino and the $Z^{0}$ boson is

$$
\Gamma\left(\widetilde{h}_{ \pm} \rightarrow \widetilde{a} Z^{0}\right) \simeq \frac{1}{32 \pi}(\cos \beta \mp \sin \beta)^{2}\left(1-\frac{m_{Z^{0}}^{2}}{m_{\widetilde{h}}^{2}}\right)^{2}\left(1+2 \frac{m_{Z^{0}}^{2}}{m_{\widetilde{h}}^{2}}\right) \frac{m_{\widetilde{h}}^{3}}{\langle\hat{S}\rangle^{2}} .
$$

The lifetime of the higgsino can be estimated as

$$
\tau_{\widetilde{h}}=\Gamma_{\widetilde{h}}^{-1} \equiv\left(\Gamma\left(\widetilde{h} \rightarrow \widetilde{a} h^{0}\right)+\Gamma\left(\widetilde{h} \rightarrow \widetilde{a} Z^{0}\right)\right)^{-1} \simeq 2.4 \times 10^{-9} \text { sec. },
$$

for $m_{h^{0}}=120 \mathrm{GeV}, m_{Z^{0}}=91 \mathrm{GeV}, m_{\widetilde{h}}=150 \mathrm{GeV}$ and $\langle S\rangle=10^{10} \mathrm{GeV}$. According to eq.(176) and eq.(81) and eq.(87), we can find that in the 2nd. process, the annihilation is more effective than the decay. Since the higgsinos decay into axinos after their annihilation, the axino abundance of the $2 n d$. process is comparable to that of the higgsinos

$$
\begin{aligned}
& \left.Y_{\widetilde{a}}^{\widetilde{h}} \simeq \frac{n_{\widetilde{h}}}{s}\right|_{T_{R}^{X}} \simeq 6.2 \times 10^{-19}\left(\frac{g_{*}\left(T_{R}^{X}\right)}{10}\right)^{-1 / 4} d_{g}^{-1}\left(\frac{m_{X}}{10^{6} \mathrm{GeV}}\right)^{-3 / 2} \frac{\mathrm{GeV}^{-2}}{\left\langle\sigma_{\text {ann. }} v_{\text {rel. }}\right\rangle_{\widetilde{h}}} \\
& \simeq 2.1 \times 10^{-11}\left(\frac{g_{*}\left(T_{R}^{X}\right)}{10}\right)^{-1 / 4} d_{g}^{-1}\left(\frac{m_{X}}{10^{6} \mathrm{GeV}}\right)^{-3 / 2} .
\end{aligned}
$$


Here, in the last step, we have set $m_{\tilde{h}}=150 \mathrm{GeV}$ for reference.

On the other hand, in the 4th. process, the annihilation does not occur effectively as in the case of the bino NLSP. Therefore, the axino abundance produced by the 4 th. process is the same as the gravitino abundance

$$
Y_{\widetilde{a}}^{\widetilde{h}} \simeq Y_{3 / 2}^{X} \simeq 4.3 \times 10^{-9}\left(\frac{g_{*}\left(T_{R}^{X}\right)}{10}\right)^{-1 / 4} d_{g}\left(\frac{N_{G}}{12}\right)^{1 / 2}\left(\frac{m_{X}}{10^{6} \mathrm{GeV}}\right)^{1 / 2},
$$

and hence we find this is the dominant contribution to the axino relic abundance. As the case of the bino NLSP, the higgsinos also become non-relativistic before its decay. The scattering rate for the higgsino is given by 64

$$
\begin{aligned}
\Gamma_{\text {scatt. }}^{\widetilde{h}} \simeq & 8 \pi^{2} \alpha_{2}^{2} \frac{E_{\widetilde{h}} T_{3 / 2}^{4}}{m_{W}^{4}} e^{-m_{\tilde{h}} \Delta m_{\tilde{\chi}} / 2 E_{\tilde{h}} T_{3 / 2}}\left(\frac{\Delta m_{\tilde{\chi}}}{m_{\tilde{h}}}+6 \frac{E_{\widetilde{h}} T_{3 / 2}}{m_{\widetilde{h}}^{2}}\right) \\
& \times\left(12 \frac{E_{\widetilde{h}} T_{3 / 2}}{m_{\widetilde{h}}^{2}}-2 \frac{\Delta m_{\tilde{\chi}}}{m_{\tilde{h}}}\right) N_{F},
\end{aligned}
$$

where $\Delta m_{\tilde{\chi}} \equiv m_{\tilde{\chi}^{+}}-m_{\tilde{h}}$ is the mass difference between the chargino and the higgsino, $E_{\widetilde{h}}$ the energy of the higgsino and $N_{F}$ the number of the processes. Taking the ratio eq.(81) to eq.(191), we find

$$
\left.\frac{\Gamma_{\text {scatt. }}^{\widetilde{h}}}{\Gamma_{\widetilde{h}}}\right|_{T_{3 / 2}} \sim 400 N_{F}
$$

for $E_{\widetilde{h}}=50 \mathrm{TeV}, m_{\tilde{h}}=150 \mathrm{GeV}, \Delta m_{\tilde{\chi}} \simeq 5 \mathrm{GeV}$ and $T_{3 / 2} \simeq 10 \mathrm{MeV}{ }^{12}$.

\subsection{Stau NLSP case}

Next, we discuss the case for the stau NLSP. The annihilation cross section of the stau is given by [65]

$$
\left\langle\sigma_{\text {ann. }} v_{\text {rel. }}\right\rangle_{\widetilde{\tau}} \simeq \frac{4 \pi \alpha_{\mathrm{em}}^{2}}{m_{\widetilde{\tau}}^{2}}+\frac{16 \pi \alpha_{\mathrm{em}}^{2} m_{\widetilde{B}}^{2}}{\cos ^{4} \theta_{W}\left(m_{\tilde{\tau}}^{2}+m_{\widetilde{B}}^{2}\right)^{2}}
$$

where $m_{\widetilde{\tau}}$ is the stau mass. For simplicity, we do not consider the coannihilation of $\widetilde{\tau}$ with $\widetilde{B}$. The stau can decay into the axino through the axino-higgsino mixing eq.(82). Thus, the coupling of the stau with the axino becomes

$$
\mathcal{L}_{\widetilde{\tau} \tau \widetilde{a}} \simeq-y_{\tau} \frac{\epsilon_{1}}{\mu} \widetilde{\tau}_{R}^{*} \tau \widetilde{a}^{\prime}+\text { h.c. }
$$

\footnotetext{
${ }^{12}$ In the other NLSP cases, one would also find the scattering process of NLSP with thermal bath is more effective than its decay width. Therefore, any NLSPs become non-relativistic before its decay.
} 
with $y_{\tau}$ the Yukawa coupling of $\tau$. Therefore, we can obtain the decay width of the stau is

$$
\Gamma(\widetilde{\tau} \rightarrow \widetilde{a} \tau) \simeq \frac{1}{32 \pi}\left(\frac{m_{\tau}}{m_{\widetilde{\tau}}}\right)^{2} \frac{m_{\widetilde{\tau}}^{3}}{\langle\hat{S}\rangle^{2}} .
$$

The lifetime is estimated as

$$
\tau_{\widetilde{\tau}} \simeq 2.0 \times 10^{-5} \text { sec. }\left(\frac{\langle\hat{S}\rangle}{10^{10} \mathrm{GeV}}\right)^{2}\left(\frac{m_{\tilde{\tau}}}{100 \mathrm{GeV}}\right)^{-1} .
$$

In the 2nd. process, since staus are produced by gauginos which are the decay product of the modulus, they are so abundant that the annihilation becomes effective. Thus, the axinos produced in the $2 n d$. process can be negligible. Comparing the decay width of the stau with the interaction rate by using eq.(93) and eq.(95), we can find that the annihilation of the stau in the 4 th. process does not become effective. Thus, when the stau is NLSP the axino abundance is also equal to the gravitino abundance,

$$
Y_{\widetilde{a}}^{\widetilde{\tau}} \simeq Y_{3 / 2}^{X} \simeq 4.3 \times 10^{-9}\left(\frac{g_{*}\left(T_{R}^{X}\right)}{10}\right)^{-1 / 4} d_{g}\left(\frac{N_{G}}{12}\right)^{1 / 2}\left(\frac{m_{X}}{10^{6} \mathrm{GeV}}\right)^{1 / 2} .
$$

\subsection{Stop and Wino NLSP cases}

We also mention the case of the stop and the wino NLSP briefly. Let us discuss first the case where the stop is the NLSP. If the stop mass is heavy enough to decay into the top quark, the decay width of the stop is obtained by (analogous to eq.(94))

$$
\Gamma(\widetilde{t} \rightarrow \widetilde{a} t) \simeq \frac{1}{32 \pi}\left(1-\frac{m_{t}^{2}}{m_{\tilde{t}}^{2}}\right)^{2}\left(\frac{m_{t}}{m_{\tilde{t}}}\right)^{2} \frac{m_{\tilde{t}}^{3}}{\langle\hat{S}\rangle^{2}},
$$

and hence the lifetime of the stop is

$$
\tau_{\tilde{t}} \simeq 1.8 \times 10^{-8} \text { sec. }\left(\frac{\langle\hat{S}\rangle}{10^{10} \mathrm{GeV}}\right)^{2}\left(\frac{m_{\tilde{t}}}{200 \mathrm{GeV}}\right)^{-1}
$$

with $m_{t}=174 \mathrm{GeV}$. The annihilation cross section of the stop is obtained by replacing the coupling constant of eq.(93) with the strong coupling,

$$
\left\langle\sigma_{\text {ann. }} v_{\text {rel. }}\right\rangle_{\tilde{t}} \simeq \frac{32 \pi \alpha_{3}^{2}}{m_{\widetilde{t}}^{2}} .
$$

Thus, when $m_{\tilde{t}} \gtrsim m_{t} \mathrm{GeV}$, we can find the annihilation process is not effective in the 4 th. process. Then, the abundance of the axino is the same as that of the other NLSP cases, i.e. eq.(70) ${ }^{13}$.

\footnotetext{
${ }^{13}$ On the other hand, if $m_{\tilde{t}} \lesssim m_{t}$, the decay width of the stop is more suppressed than eq. (98) by the three-body phase space. In such a case, since the annihilation may become effective, the axino abundance in the 4 th. process is much smaller than that of the case. Therefore, when $m_{\tilde{t}} \lesssim m_{t}$ the axino cannot explain the present DM abundance.
} 
If the wino is NLSP ${ }^{14}$, its decay width can be obtained by the analogy of that of the bino:

$$
\begin{gathered}
\Gamma(\widetilde{W} \rightarrow \widetilde{a} \gamma) \simeq \frac{\alpha_{\mathrm{em}}^{2} N^{2}}{256 \pi^{3}} \frac{1}{\sin ^{2} \theta_{W}} \frac{m_{\widetilde{W}}^{3}}{\langle\hat{S}\rangle^{2}} \\
\Gamma\left(\widetilde{W} \rightarrow \widetilde{a} Z^{0}\right) \simeq \frac{\alpha_{\mathrm{em}}^{2} N^{2}}{256 \pi^{3}} \frac{\cos ^{2} \theta_{W}}{\sin ^{4} \theta_{W}}\left(1-x_{Z}\right)\left(1-\frac{x_{Z}}{2}-\frac{x_{Z}^{2}}{2}\right) \frac{m_{\widetilde{W}}^{3}}{\langle\hat{S}\rangle^{2}},
\end{gathered}
$$

where $m_{\widetilde{W}}$ is the wino mass. For $m_{\widetilde{W}}=100 \mathrm{GeV}$, the lifetime of the wino is

$$
\begin{aligned}
\tau_{\widetilde{W}} & =\Gamma_{\widetilde{W}}^{-1} \equiv\left(\Gamma(\widetilde{W} \rightarrow \widetilde{a} \gamma)+\Gamma\left(\widetilde{W} \rightarrow \widetilde{a} Z^{0}\right)\right)^{-1} \\
& \simeq 1.9 \times 10^{-3} \text { sec.. }
\end{aligned}
$$

On the other hand, it is known that the annihilation of the wino pair is very effective. The annihilation cross section is given by 61 ]

$$
\left\langle\sigma_{\text {ann. }} v_{\text {rel. }}\right\rangle_{\widetilde{W}}=\frac{8 \pi \alpha_{2}^{2}}{m_{\widetilde{W}}^{2}} \frac{\left(1-x_{W}\right)^{3 / 2}}{\left(2-x_{W}\right)^{2}} .
$$

Then we can find that when $m_{\widetilde{W}} \simeq 100 \mathrm{GeV},\left\langle\sigma_{\text {ann. }} v_{\text {rel. }}\right\rangle_{\widetilde{W}} n_{\widetilde{W}} \gg \Gamma_{\widetilde{W}}$ at the gravitino decay, where $n_{\widetilde{W}}$ is the number density of the wino. Thus the abundance of the axino is the same as that of the wino after annihilation,

$$
\left.Y_{\widetilde{a}}^{\widetilde{W}} \simeq \frac{n_{\widetilde{W}}}{s}\right|_{T_{3 / 2}} \simeq 3.3 \times 10^{-11}\left(\frac{g_{*}\left(T_{3 / 2}\right)}{10}\right)^{-1 / 4}\left(\frac{m_{3 / 2}}{10^{5} \mathrm{GeV}}\right)^{-3 / 2}\left(\frac{m_{\widetilde{W}}}{100 \mathrm{GeV}}\right)^{2}
$$

where we have used $x_{W} \simeq 0.64$ evaluated by $m_{\widetilde{W}}=100 \mathrm{GeV}$. On the other hand, when $m_{\widetilde{W}} \gtrsim$ $300 \mathrm{GeV}$, the annihilation process does not occur effectively, and then the axino abundance is equal to that of the other NLSP cases.

Here, we summarize the axino abundance for each NLSP cases in table.1, where we showed only the 4 th. process because the other processes are subdominant. When $\widetilde{B}, \widetilde{h}$ and $\widetilde{\tau}$ are NLSP cases, the annihilation is always less effective than the decay. Since the annihilation cross section of the stop is quite large, the axino abundance becomes too small to explain the present DM abundance when the annihilation of the stop is effective.

\section{$6 \quad$ Results}

At last, we are now ready to discuss whether the axino relic abundance can explain the present DM abundance. If the decay of NLSP is more effective than the annihilation in the 4 th. process,

\footnotetext{
${ }^{14}$ This does not occur for $c_{\lambda_{a}}=1$ and $\alpha \simeq 1$. However, once we introduce e.g. non-universal $c_{\tilde{B}}$ 67-69, it is possible to have wino NLSP, which will not upset the stabilization mechanism of $S$ controlled by the strong interaction unless bino is hierarchically heavy.
} 


\begin{tabular}{|c|c|c|}
\hline NLSP & Case A: $\Gamma \gg n\langle\sigma v\rangle$ & Case B: $\Gamma \ll n\langle\sigma v\rangle$ \\
\hline$\widetilde{B}$ & \multirow{5}{*}{$Y_{\widetilde{a}} \simeq 4.3 \times 10^{-9}$} & \multirow{3}{*}{ does not occur } \\
\hline$\widetilde{h}$ & & \\
\hline$\widetilde{\tau}$ & & \\
\hline$\widetilde{t}$ & & too small abundance \\
\hline$\widetilde{W}$ & & $Y_{\widetilde{a}} \simeq 3.3 \times 10^{-11}$ \\
\hline
\end{tabular}

Table 1: Table of abundance of the axino in the 4th. process. Contributions of the other processes are subdominant. Cases A and B, respectively, correspond to situations where the annihilation process of NLSP is not effective and effective.

the $\Omega$ parameter, which is defined by the ratio of the mass density of $\widetilde{a}$ and the critical density, is the same in any NLSP cases

$$
\Omega_{\widetilde{a}} h^{2} \simeq 1.2\left(\frac{m_{\widetilde{a}}}{1 \mathrm{GeV}}\right)\left(\frac{g_{*}\left(T_{R}^{X}\right)}{10}\right)^{-1 / 4} d_{g}\left(\frac{N_{G}}{12}\right)^{1 / 2}\left(\frac{m_{X}}{10^{6} \mathrm{GeV}}\right)^{1 / 2} .
$$

On the other hand, if the wino is NLSP and their annihilation is effective, the $\Omega$ parameter is

$$
\begin{aligned}
\Omega_{\widetilde{a}} h^{2} \simeq 10^{-3}\left(\frac{m_{\widetilde{a}}}{1 \mathrm{GeV}}\right)\{ & 26\left(\frac{g_{*}\left(T_{3 / 2}\right)}{10}\right)^{-1 / 4}\left(\frac{m_{3 / 2}}{50 \mathrm{TeV}}\right)^{-3 / 2}\left(\frac{m_{\widetilde{W}}}{100 \mathrm{GeV}}\right)^{2} \\
& \left.+\left(4.8+1.5 \lambda^{4} N^{2} k^{2}\right)\left(\frac{g_{*}\left(T_{R}^{X}\right)}{10}\right)^{-1 / 4}\left(\frac{m_{X}}{10^{6} \mathrm{GeV}}\right)^{1 / 2}\right\} .
\end{aligned}
$$

Here we have set $d_{g}=1$ and $N_{G}=12$. In fig, 4, we plotted eq.(105) in terms of $m_{X}$ and $m_{\tilde{a}}$. From the WMAP three year results [70], the DM abundance in the present Universe is $\Omega_{\mathrm{DM}} h^{2}=0.105_{-0.013}^{+0.007}\left(68 \%\right.$ C.L.). Thus, we can find from fig, 4 that the axino with $m_{\widetilde{a}} \simeq \mathcal{O}(100)$ $\mathrm{MeV}$ can explain the present DM abundance in any NLSP cases, if the decay of NLSP is more effective than the annihilation in the 4 th. process. In fig.5, we have plotted axino mass contours which satisfy $\Omega_{\widetilde{a}} h^{2}=0.1$ in $m_{3 / 2}-m_{X}$ plane for the wino NLSP case, where we set $\lambda=N=1$, $k=0$, and $m_{\widetilde{W}}=100 \mathrm{GeV}$. If the wino is NLSP and their annihilation is effective, fig.5 shows that the right amount of DM can be explained by the axino with a few $\mathrm{GeV}$ mass ${ }^{15}$. Here we should caution that the estimate of the relic abundance given in this paper is rather rough, which may contain an error of factor 2 or so. From eq.(25), we can parametrize the axino mass by using $\lambda$ as

$$
m_{\widetilde{a}}=\frac{\alpha_{3} \lambda^{2}}{4 \pi^{3}} F_{\Phi} .
$$

\footnotetext{
${ }^{15}$ Here, we should consider that the gravitino mass is heavier than about $30 \mathrm{TeV}$ not to spoil the success of BBN [1]- 73 .
} 
Substituting eq.(107) into $\Omega$ parameters, we can find

$$
\Omega_{\widetilde{a}} h^{2} \simeq 0.1\left(\frac{\lambda}{0.06}\right)^{2}\left(\frac{F_{\Phi}}{50 \mathrm{TeV}}\right)\left(\frac{m_{X}}{10^{6} \mathrm{GeV}}\right)^{1 / 2}\left(\frac{g_{*}\left(T_{R}^{X}\right)}{10}\right)^{-1 / 4} d_{g}\left(\frac{N_{G}}{12}\right)^{1 / 2},
$$

for the case where the annihilation is not effective, and

$$
\begin{aligned}
\Omega_{\widetilde{a}} h^{2} \simeq\left(\frac{\lambda}{0.4}\right)^{2}\left(\frac{F_{\Phi}}{50 \mathrm{TeV}}\right)\{ & 0.09\left(\frac{g_{*}\left(T_{3 / 2}\right)}{10}\right)^{-1 / 4}\left(\frac{m_{3 / 2}}{50 \mathrm{TeV}}\right)^{-3 / 2}\left(\frac{m_{\widetilde{W}}}{100 \mathrm{GeV}}\right)^{2} \\
& \left.+0.02\left(\frac{g_{*}\left(T_{R}^{X}\right)}{10}\right)^{-1 / 4}\left(\frac{m_{X}}{10^{6} \mathrm{GeV}}\right)^{1 / 2}\right\},
\end{aligned}
$$

for the wino NLSP case, whose annihilation process is effective. Thus, $\mathcal{O}(0.1)$ coupling between the axion superfield and messengers leads to the right amount of DM in any NLSP cases.

Here, we should mention the free-streaming scale of the axino LSP. According to the discussion in the previous section, the axino LSPs are produced mainly at the decays of non-relativistic NLSPs. At the production, they are relativistic and travel freely until their momentum gets redshifted to be non-relativistic. The observations of Lyman- $\alpha$ forest require that the free-streaming scale of DM have to be less than $\mathcal{O}(1) \mathrm{Mpc}$ [74] [75]. We can estimate the free-streaming scale of the axino as [76] [77]

$$
\lambda_{\mathrm{FS}}=\int_{t_{\widetilde{a}}}^{t_{\mathrm{EQ}}} \frac{v(t)}{a(t)} d t \simeq 1.0 \mathrm{Mpc} u_{\widetilde{a}}\left[\frac{t_{\widetilde{a}}}{10^{6} \mathrm{~s}}\right]^{1 / 2}\left\{1+0.14 \ln \left[\left(\frac{10^{6} \mathrm{~s}}{t_{\widetilde{a}}}\right)^{1 / 2} \frac{1}{u_{\widetilde{a}}}\right]\right\},
$$

where $v(t)$ and $a(t)$ are the velocity of the axino and the scale factor, respectively. $t_{\mathrm{EQ}}$ is the time at matter-radiation equality, $t_{\widetilde{a}}$ the time when the axino is produced and the three-momentum normalized by the axino mass $u_{\widetilde{a}}=p / m_{\widetilde{a}}$. From the discussion of section 5, we can approximate that $t_{\widetilde{a}}$ is equal to the lifetime of the gravitino, and hence we find

$$
\lambda_{\mathrm{FS}} \simeq 0.4 \mathrm{Mpc}\left(\frac{m_{\mathrm{NLSP}}}{200 \mathrm{GeV}}\right)\left(\frac{m_{\widetilde{a}}}{0.1 \mathrm{GeV}}\right)^{-1}\left(\frac{m_{3 / 2}}{50 \mathrm{TeV}}\right)^{-3 / 2},
$$

for any NLSP cases if the annihilation process is not effective. The axinos produced directly by the decays of moduli and gravitinos have much larger free-streaming length of order $10 \mathrm{Mpc}$. However, their contamination in the total dark matter density is about $1 \%$ level, so that their contribution will be harmless [74.

On the other hand, in the case where the annihilation process is effective and NLSP is the wino with $m_{\widetilde{W}}=100 \mathrm{GeV}$, axinos produced by gravitinos and the modulus have the free-streaming scale of the order of $\mathcal{O}(1) \mathrm{Mpc}^{16}$ :

$$
\begin{gathered}
\lambda_{\mathrm{FS}} \simeq 1.3 \mathrm{Mpc}\left(\frac{m_{3 / 2}}{10^{5} \mathrm{GeV}}\right)^{-1 / 2}\left(\frac{m_{\widetilde{a}}}{5 \mathrm{GeV}}\right)^{-1}, \\
\lambda_{\mathrm{FS}} \simeq 0.4 \mathrm{Mpc}\left(\frac{m_{X}}{5 \times 10^{6} \mathrm{GeV}}\right)^{-1 / 2}\left(\frac{m_{\widetilde{a}}}{5 \mathrm{GeV}}\right)^{-1} .
\end{gathered}
$$

\footnotetext{
${ }^{16}$ The free-streaming scale of the axino produced by the stau or the wino NLSP is $\mathcal{O}(0.01)$ Mpc.
} 
The free-streaming scales of the axino, eq.(111) and eq.(112), are in an interesting range for the small structure problem point of view. The model of the cold dark matter (CDM) can explain the large scale structure of the Universe very well. However, $N$-body simulations in the CDM model seem to conflict with observations at the smaller scale $(\lesssim \mathrm{Mpc})$. Numerical simulations based on the CDM model predict that the number of halos is by one order of magnitude larger than observations within $\mathcal{O}(1) \mathrm{Mpc}$. This is called the missing satellite problem [78]. Another possible discrepancy is that the prediction of simulations for mass profile of CDM halos is excessively cuspy compared with observations, which is known as the cusp problem [79]. One of the resolutions to these problems is to invoke some DM whose free-streaming scale is in the range of $\mathcal{O}(0.1-1)$ Mpc, because it can sweep out density fluctuations in the small scale [80]. Then the axino LSP of our model may also be able to solve these problems.

Before concluding this paper, we also mention whether the axion can explain DM. We have already discussed that the singlet, $S$, can be stabilized at $10^{10} \mathrm{GeV}$ to $10^{12} \mathrm{GeV}$ by choosing the modular weight of the messenger appropriately. The axion relic abundance eq.(22) with $\Theta=\mathcal{O}(1)$ shows that when the PQ scale is obtained $f_{\mathrm{PQ}} \simeq 10^{12} \mathrm{GeV}$ the axion can constitute DM abundance. When the axion superfield is stabilized at $10^{12} \mathrm{GeV}$, however, the lifetime of the saxion is longer than that of the modulus, then the Universe may be dominated by the saxion if the initial amplitude of the saxion is not so small compared with the Planck scale. Since the saxion mainly decays into axions, it will spoil the success of BBN. Therefore, to explain DM by the axion would be difficult in our model unless the initial amplitude of the saxion is suppressed or there is an extra entropy production.

\section{Summary}

Let us summarize this paper. We have discussed the axionic extended mirage mediation, axionic mirage mediation, to remedy crucial problems included in the mirage mediation: the $\mu$-problem and the moduli problem. We showed that the PQ scale can be obtained in the axion window, $10^{9} \mathrm{GeV} \lesssim f_{\mathrm{PQ}} \lesssim 10^{12-13} \mathrm{GeV}$, by stabilizing the axion superfield. Especially, in the KKLT set-up $(\alpha \simeq 1)$ with $\ell=1 / 2$, we obtained the PQ scale at $10^{10} \mathrm{GeV}$. To obtain desired values of $\mu$ - $/ B \mu$-term was achieved by introducing some singlets with couplings eq.(26) and eq.(27) and integrating out the singlet $T$ which becomes heavy due to the $\mathrm{VEV}$ of the axion superfield. It was also found that the phase of the $B$-parameter can be rotated away simultaneously with the phases of the gaugino mass and the $A$-parameter. Thus, SUSY CP problem is also absent in our model.

We investigated the implications of the modulus, whose mass is larger than that of the gravitino by several order of magnitude, and the saxion to cosmology. When both the saxion and the modulus have the order of the Planck scale initial amplitude, the Universe is dominated by the oscillation energy of the modulus eventually. In this case, we found that as long as the saxion lifetime is sufficiently shorter than that of the modulus, axions produced by the saxion decay would not spoil the success of BBN. Thus, we discussed the cosmic evolution only after 
the modulus decay. Although there were several axino LSP production processes, the dominant contribution to the axino relic abundance came from the decay of the NLSPs produced by the gravitinos. We have estimated the axino abundance for the various NLSP cases. However, we found that the axino relic abundance is the same in any NLSP cases, if the decay width of NLSP into the axino is larger than their annihilation rate. In such a situation, we found that the axino mass with $\mathcal{O}(100) \mathrm{MeV}$ leads to the present $\mathrm{DM}$ abundance. On the other hand, in the case where NLSP is the wino and their interaction rate is more effective than their decay process, the axino abundance is less than the former case about two orders. In this case, the DM abundance can be explained by the axino with $m_{\tilde{a}} \simeq \mathcal{O}(1) \mathrm{GeV}$. When we parametrize the axino mass in terms of the Yukawa coupling of $S, \lambda$, by eq.(107), $\lambda \simeq \mathcal{O}(0.1)$ explains the right amount of DM abundance, eq.(108) and eq.(109). Thus, nevertheless the branching ratio of the modulus to the gravitino pair is sizable, our model can solve the moduli problem as well as leads to right amount of DM abundance. In addition, we also found that the free-streaming scale of the axino LSP is in an interesting region $\mathcal{O}(0.1-1) \mathrm{Mpc}$ for the small structure problems.

Finally we would like to briefly mention signatures of neutralino NLSP decays at collider experiments. As was discussed in this paper, the axino LSP couples to the Higgs multiplets at tree level, whereas its coupling to the gauge sector arises at one loop, and thus is suppressed. This implies that the neutralino NLSP dominantly decays to the Higgs boson (as well as the $Z^{0}$ boson) as far as it contains a sizable fraction of the higgsino components. We expect that the NLSP decay into the Higgs boson will provide a spectacular signal of displaced vertex emitting hard jets, if the decay vertex can be reconstructed. Detailed study on the collider signatures will be presented elsewhere.

\section{Acknowledgement}

We would like to thank T. Higaki and A. Yotsuyanagi for useful discussions. The work was partially supported by the grants-in-aid from the Ministry of Education, Science, Sports, and Culture of Japan, No.16081202 and No.17340062. K.O. is supported by the Grand-in-aid for Scientific Research No.19740144 from the Ministry of Education, Culture, Sports, Science and

Technology of Japan. K.O. also thanks Yukawa Institute in Kyoto University for the use of Altix3700 BX2.

\section{References}

[1] V. S. Kaplunovsky and J. Louis, Phys. Lett. B 306, 269 (1993) arXiv:hep-th/9303040]; A. Brignole, L. E. Ibanez and C. Munoz, Nucl. Phys. B 422, 125 (1994) [Erratum-ibid. B 436, 747 (1995)] arXiv:hep-ph/9308271.

[2] L. Randall and R. Sundrum, Nucl. Phys. B 557, 79 (1999) arXiv:hep-th/9810155. 
[3] G. F. Giudice, M. A. Luty, H. Murayama and R. Rattazzi, JHEP 9812, 027 (1998) arXiv:hep-ph/9810442.

[4] J. A. Bagger, T. Moroi and E. Poppitz, JHEP 0004, 009 (2000) arXiv:hep-th/9911029.

[5] S. Kachru, R. Kallosh, A. Linde and S. P. Trivedi, Phys. Rev. D 68, 046005 (2003) [arXiv:hep-th/0301240].

[6] K. Choi, A. Falkowski, H. P. Nilles, M. Olechowski and S. Pokorski, JHEP 0411, 076 (2004) arXiv:hep-th/0411066.

[7] K. Kohri, M. Yamaguchi and J. Yokoyama, Phys. Rev. D 72, 083510 (2005) arXiv:hep-ph/0502211].

[8] K. Choi, A. Falkowski, H. P. Nilles and M. Olechowski, Nucl. Phys. B 718, 113 (2005) arXiv:hep-th/0503216.

[9] M. Endo, M. Yamaguchi and K. Yoshioka, Phys. Rev. D 72, 015004 (2005) arXiv:hep-ph/0504036].

[10] K. Choi, K. S. Jeong and K. i. Okumura, JHEP 0509, 039 (2005) arXiv:hep-ph/0504037.

[11] A. Falkowski, O. Lebedev and Y. Mambrini, JHEP 0511, 034 (2005) arXiv:hep-ph/0507110].

[12] H. Baer, E. K. Park, X. Tata and T. T. Wang, JHEP 0608, 041 (2006) arXiv:hep-ph/0604253.

[13] K. Kawagoe and M. M. Nojiri, Phys. Rev. D 74, 115011 (2006) arXiv:hep-ph/0606104].

[14] K. Choi, K. Y. Lee, Y. Shimizu, Y. G. Kim and K. i. Okumura, JCAP 0612, 017 (2006) arXiv:hep-ph/0609132.

[15] M. Nagai and K. Nakayama, Phys. Rev. D 76, 123501 (2007) arXiv:0709.3918 [hep-ph]].

[16] B. de Carlos, J. A. Casas, F. Quevedo and E. Roulet, Phys. Lett. B 318, 447 (1993) |arXiv:hep-ph/9308325|. T. Banks, D. B. Kaplan and A. E. Nelson, Phys. Rev. D 49, 779 (1994) |arXiv:hep-ph/9308292].

[17] M. Endo, K. Hamaguchi and F. Takahashi, Phys. Rev. Lett. 96, 211301 (2006) arXiv:hep-ph/0602061].

[18] S. Nakamura and M. Yamaguchi, Phys. Lett. B 638, 389 (2006) arXiv:hep-ph/0602081.

[19] T. Asaka, S. Nakamura and M. Yamaguchi, Phys. Rev. D 74, 023520 (2006) arXiv:hep-ph/0604132. 
[20] M. Kawasaki, F. Takahashi and T. T. Yanagida, Phys. Lett. B 638, 8 (2006) arXiv:hep-ph/0603265.

[21] M. Ibe, Y. Shinbara and T. T. Yanagida, Phys. Lett. B 639, 534 (2006) arXiv:hep-ph/0605252.

[22] S. Nakamura and M. Yamaguchi, Phys. Lett. B 655, 167 (2007) [arXiv:0707.4538 [hep-ph]].

[23] R. D. Peccei and H. R. Quinn, Phys. Rev. Lett. 38, 1440 (1977).

[24] F. Wilczek, Phys. Rev. Lett. 40, 279 (1978).

[25] J. E. Kim, Phys. Rev. Lett. 43, 103 (1979); M. A. Shifman, A. I. Vainshtein and V. I. Zakharov, Nucl. Phys. B 166, 493 (1980).

[26] M. Dine, W. Fischler and M. Srednicki, Phys. Lett. B 104, 199 (1981); A. R. Zhitnitsky, Sov. J. Nucl. Phys. 31 (1980) 260 [Yad. Fiz. 31 (1980) 497].

[27] K. Rajagopal, M. S. Turner and F. Wilczek, Nucl. Phys. B 358, 447 (1991).

[28] T. Goto and M. Yamaguchi, Phys. Lett. B 276, 103 (1992).

[29] T. Asaka and M. Yamaguchi, Phys. Lett. B 437, 51 (1998) arXiv:hep-ph/9805449]; Phys. Rev. D 59, 125003 (1999) arXiv:hep-ph/9811451.

[30] N. Abe, T. Moroi and M. Yamaguchi, JHEP 0201, 010 (2002) arXiv:hep-ph/0111155].

[31] M. Kawasaki, K. Nakayama and M. Senami, arXiv:0711.3083 [hep-ph].

[32] K. Choi and K. S. Jeong, JHEP 0701, 103 (2007) [arXiv:hep-th/0611279].

[33] T. Banks, M. Dine and M. Graesser, Phys. Rev. D 68, 075011 (2003) [arXiv:hep-ph/0210256].

[34] E. J. Chun, J. E. Kim and H. P. Nilles, Phys. Lett. B 287, 123 (1992) arXiv:hep-ph/9205229;

[35] E. J. Chun, H. B. Kim and J. E. Kim, Phys. Rev. Lett. 72, 1956 (1994) arXiv:hep-ph/9305208;

[36] L. Covi, J. E. Kim and L. Roszkowski, Phys. Rev. Lett. 82, 4180 (1999) arXiv:hep-ph/9905212;

[37] L. Covi, H. B. Kim, J. E. Kim and L. Roszkowski, JHEP 0105, 033 (2001) arXiv:hep-ph/0101009.

[38] T. Asaka and M. Yamaguchi, unpublished.

[39] A. Pomarol and R. Rattazzi, JHEP 9905, 013 (1999) arXiv:hep-ph/9903448]; R. Rattazzi, A. Strumia and J. D. Wells, Nucl. Phys. B 576, 3 (2000) |arXiv:hep-ph/9912390|. 
[40] O. Lebedev, H. P. Nilles and M. Ratz, Phys. Lett. B 636, 126 (2006) arXiv:hep-th/0603047.

[41] A. Pierce and J. Thaler, JHEP 0609, 017 (2006) arXiv:hep-ph/0604192].

[42] E. Dudas, C. Papineau and S. Pokorski, JHEP 0702, 028 (2007) [arXiv:hep-th/0610297].

[43] H. Abe, T. Higaki, T. Kobayashi and Y. Omura, Phys. Rev. D 75, 025019 (2007) arXiv:hep-th/0611024].

[44] M. Endo, M. Yamaguchi and K. Yoshioka, Phys. Lett. B 586, 382 (2004) [arXiv:hep-ph/0311206].

[45] K. Choi, Phys. Rev. Lett. 72, 1592 (1994) arXiv:hep-ph/9311352.

[46] K. Choi and K. S. Jeong, JHEP 0608, 007 (2006) [arXiv:hep-th/0605108].

[47] A. Anisimov, M. Dine, M. Graesser and S. D. Thomas, Phys. Rev. D 65, 105011 (2002) arXiv:hep-th/0111235.

[48] A. Anisimov, M. Dine, M. Graesser and S. D. Thomas, JHEP 0203, 036 (2002) arXiv:hep-th/0201256].

[49] F. Brummer, A. Hebecker and M. Trapletti, Nucl. Phys. B 755, 186 (2006) arXiv:hep-th/0605232.

[50] S. Kachru, L. McAllister and R. Sundrum, JHEP 0710, 013 (2007) arXiv:hep-th/0703105.

[51] H. Abe, T. Higaki and T. Kobayashi, Phys. Rev. D 73, 046005 (2006) arXiv:hep-th/0511160].

[52] W. M. Yao et al. [Particle Data Group], J. Phys. G 33 (2006) 1.

[53] H. T. Janka, W. Keil, G. Raffelt and D. Seckel, Phys. Rev. Lett. 76, 2621 (1996) arXiv:astro-ph/9507023.

[54] K. Choi, K. S. Jeong, T. Kobayashi and K. i. Okumura, Phys. Lett. B 633, 355 (2006) arXiv:hep-ph/0508029].

[55] R. Kitano and Y. Nomura, Phys. Lett. B 631, 58 (2005) arXiv:hep-ph/0509039].

[56] K. Choi, K. S. Jeong, T. Kobayashi and K. i. Okumura, Phys. Rev. D 75, 095012 (2007) arXiv:hep-ph/0612258.

[57] G. F. Giudice and A. Masiero, Phys. Lett. B 206, 480 (1988).

[58] D. H. Lyth and E. D. Stewart, Phys. Rev. D 53, 1784 (1996) [arXiv:hep-ph/9510204].

[59] M. Dine, R. Kitano, A. Morisse and Y. Shirman, Phys. Rev. D 73, 123518 (2006) arXiv:hep-ph/0604140]. 
[60] M. Endo, K. Hamaguchi and F. Takahashi, Phys. Rev. D 74, 023531 (2006) arXiv:hep-ph/0605091.

[61] K. A. Olive and M. Srednicki, Phys. Lett. B 230, 78 (1989); Nucl. Phys. B 355, 208 (1991).

[62] T. Moroi, PhD thesis, arXiv:hep-ph/9503210.

[63] M. Kawasaki, T. Moroi and T. Yanagida, Phys. Lett. B 370, 52 (1996) arXiv:hep-ph/9509399.

[64] J. Hisano, K. Kohri and M. M. Nojiri, Phys. Lett. B 505, 169 (2001) arXiv:hep-ph/0011216.

[65] T. Asaka, K. Hamaguchi and K. Suzuki, Phys. Lett. B 490, 136 (2000) arXiv:hep-ph/0005136.

[66] T. Moroi and L. Randall, Nucl. Phys. B 570, 455 (2000) arXiv:hep-ph/9906527

[67] H. Abe, Y. G. Kim, T. Kobayashi and Y. Shimizu, JHEP 0709, 107 (2007) arXiv:0706.4349 [hep-ph]].

[68] H. Verlinde, L. T. Wang, M. Wijnholt and I. Yavin, arXiv:0711.3214 [hep-th].

[69] R. Dermisek, H. Verlinde and L. T. Wang, arXiv:0711.3211 [hep-ph].

[70] D. N. Spergel et al. [WMAP Collaboration], Astrophys. J. Suppl. 170, 377 (2007) arXiv:astro-ph/0603449].

[71] M. Kawasaki, K. Kohri and T. Moroi, Phys. Lett. B 625, 7 (2005) arXiv:astro-ph/0402490.

[72] M. Kawasaki, K. Kohri and T. Moroi, Phys. Rev. D 71, 083502 (2005) arXiv:astro-ph/0408426.

[73] K. Kohri, T. Moroi and A. Yotsuyanagi, Phys. Rev. D 73, 123511 (2006) arXiv:hep-ph/0507245.

[74] M. Viel, J. Lesgourgues, M. G. Haehnelt, S. Matarrese and A. Riotto, Phys. Rev. D 71, 063534 (2005) arXiv:astro-ph/0501562].

[75] V. K. Narayanan, D. N. Spergel, R. Dave and C. P. Ma, arXiv:astro-ph/0005095.

[76] S. Borgani, A. Masiero and M. Yamaguchi, Phys. Lett. B 386, 189 (1996) arXiv:hep-ph/9605222.

[77] J. A. R. Cembranos, J. L. Feng, A. Rajaraman and F. Takayama, Phys. Rev. Lett. 95, 181301 (2005) arXiv:hep-ph/0507150. 
[78] A. A. Klypin, A. V. Kravtsov, O. Valenzuela and F. Prada, Astrophys. J. 522, 82 (1999) arXiv:astro-ph/9901240]; B. Moore, S. Ghigna, F. Governato, G. Lake, T. Quinn, J. Stadel and P. Tozzi, Astrophys. J. 524 (1999) L19; A. R. Zentner and J. S. Bullock, Astrophys. J. 598, 49 (2003) arXiv:astro-ph/0304292.

[79] R. A. Flores and J. R. Primack, Astrophys. J. 427, L1 (1994) arXiv:astro-ph/9402004; W. J. G. de Blok and S. S. McGaugh, Mon. Not. Roy. Astron. Soc. 290, 533 (1997) arXiv:astro-ph/9704274|; J. J. Binney and N. W. Evans, Mon. Not. Roy. Astron. Soc. 327, L27 (2001) [arXiv:astro-ph/0108505]; A. R. Zentner and J. S. Bullock, Phys. Rev. D 66, 043003 (2002) |arXiv:astro-ph/0205216]; J. D. Simon, A. D. Bolatto, A. Leroy, L. Blitz and E. L. Gates, Astrophys. J. 621, 757 (2005) [arXiv:astro-ph/0412035].

[80] W. B. Lin, D. H. Huang, X. Zhang and R. H. Brandenberger, Phys. Rev. Lett. 86, 954 (2001) arXiv:astro-ph/0009003. 


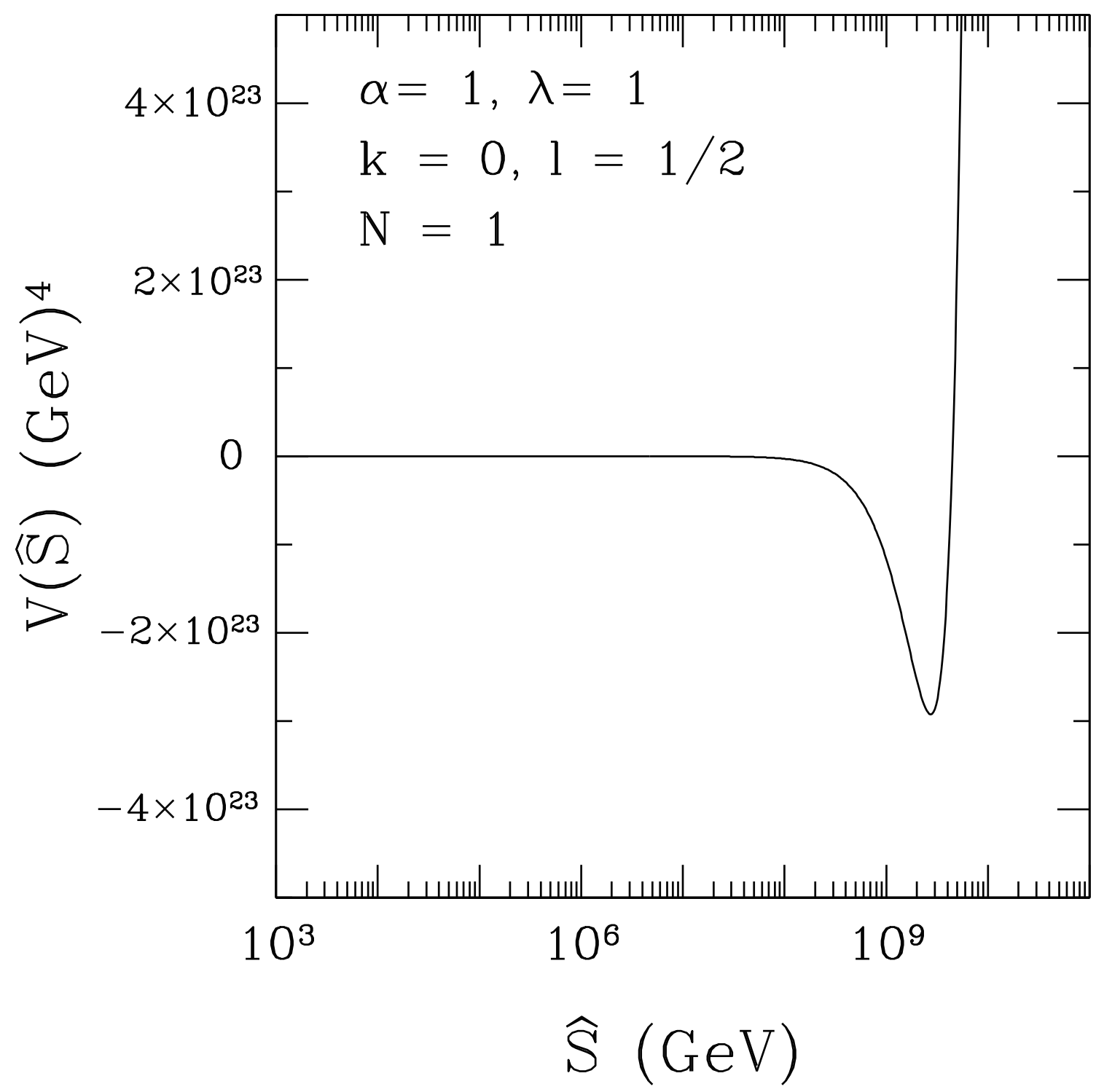

Figure 1: The saxion potential in the axionic mirage mediation. The minimal model, $f \propto X$ $\left(c_{\lambda_{a}}=1\right)$ is considered with $\alpha=1, k=0, \ell=1 / 2, \lambda=1$, and $N=1$, which satisfies the mirage condition. 


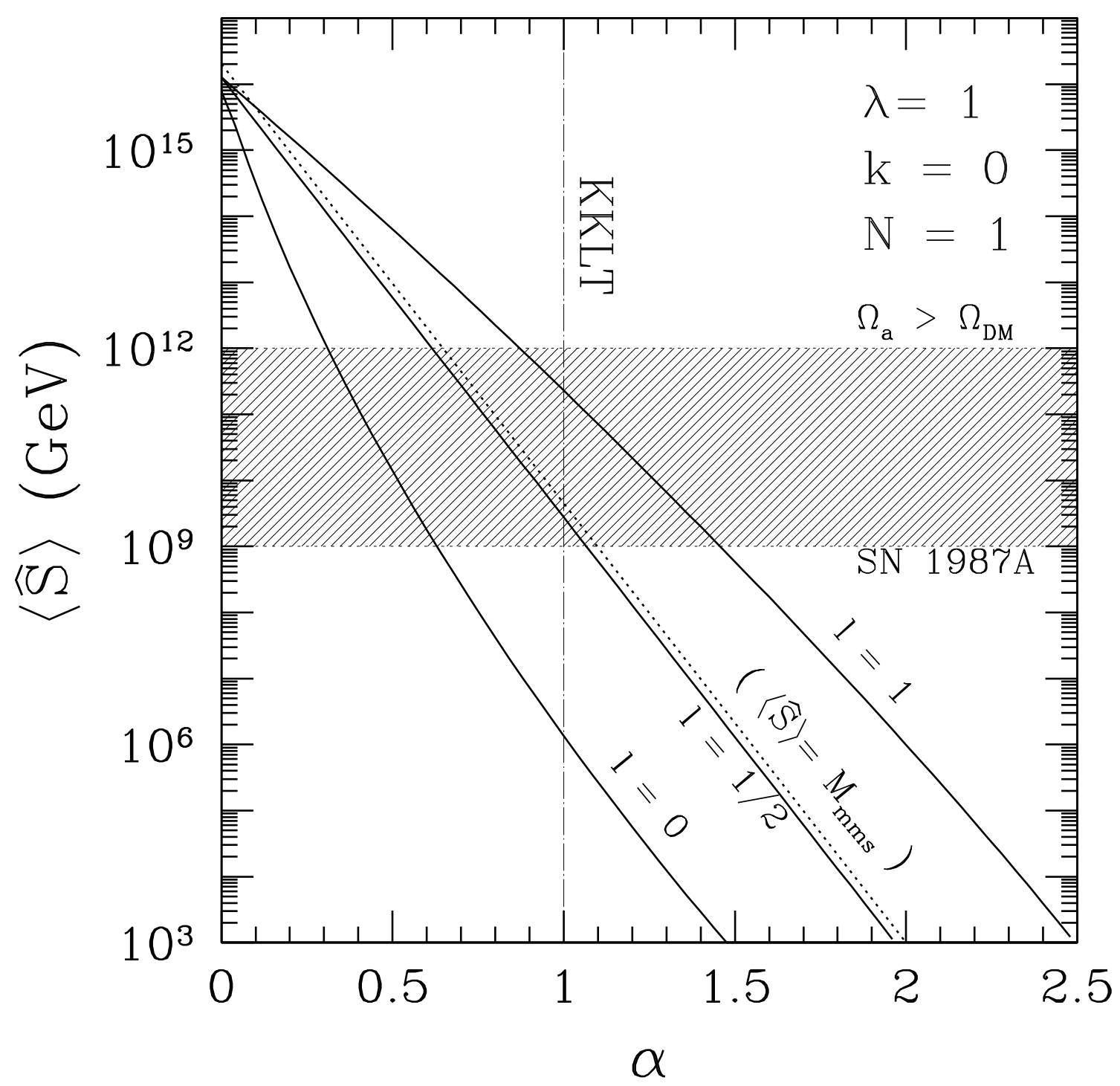

Figure 2: The PQ scale $f_{\mathrm{PQ}} \simeq\langle\hat{S}\rangle$ in the axionic mirage mediation. The minimal model, $f_{a} \propto X$ $\left(c_{\lambda_{a}}=1\right)$ is assumed. Three different cases, $\ell=0,1 / 2,1$ are shown as a function of $\alpha$ for $k=0$. $M_{m m s}$ is the mirage messenger scale at which $m_{S}^{2}(\ell=1 / 2)$ crosses zero. The remaining parameters are fixed to $\lambda=1$ and $N=1$, for which $f_{\mathrm{PQ}}$ shows quite modest dependence. For $k>0, f_{\mathrm{PQ}}$ is considerably lowered than depicted in small $\lambda$ region $(\lesssim 1)$ due to the modulous contribution to $m_{S}^{2}$. 


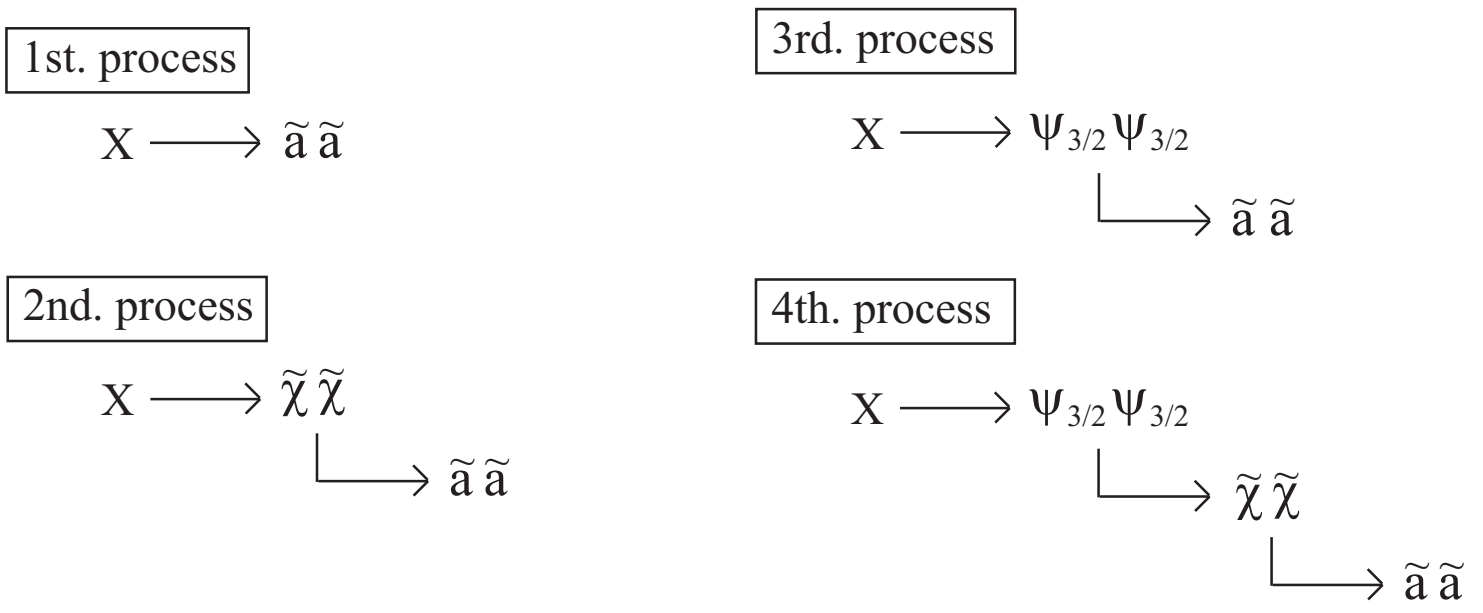

Figure 3: The sketch of four production processes of the axino, where $\tilde{\chi}$ denotes NLSP. The 4 th. process gives the dominat contribution to the relice abundance of the axino.

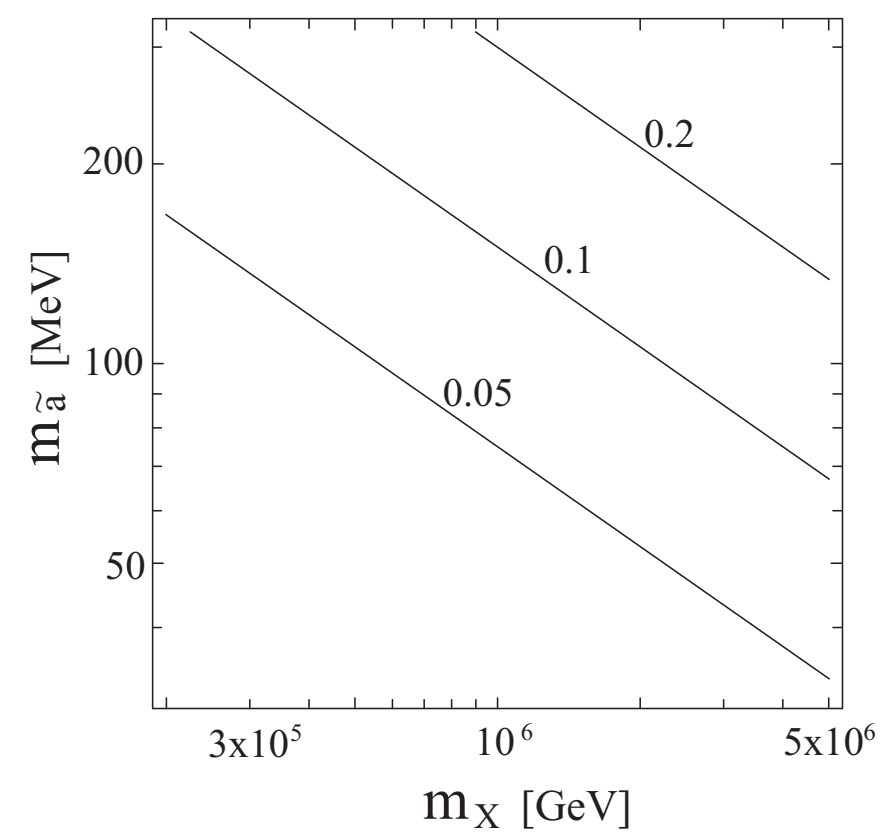

Figure 4: Contours of the density parameter of the axino, $\Omega_{\widetilde{a}} h^{2}$, drawn in $m_{X}-m_{\widetilde{a}}$ plane. Three lines represent $\Omega_{\widetilde{a}} h^{2}=0.2,0.1$ and 0.05 , from the above. The contours are the same in any NLSP cases if the decay of NLSP produced by the gravitino decay is more effective than the annihilation process. 


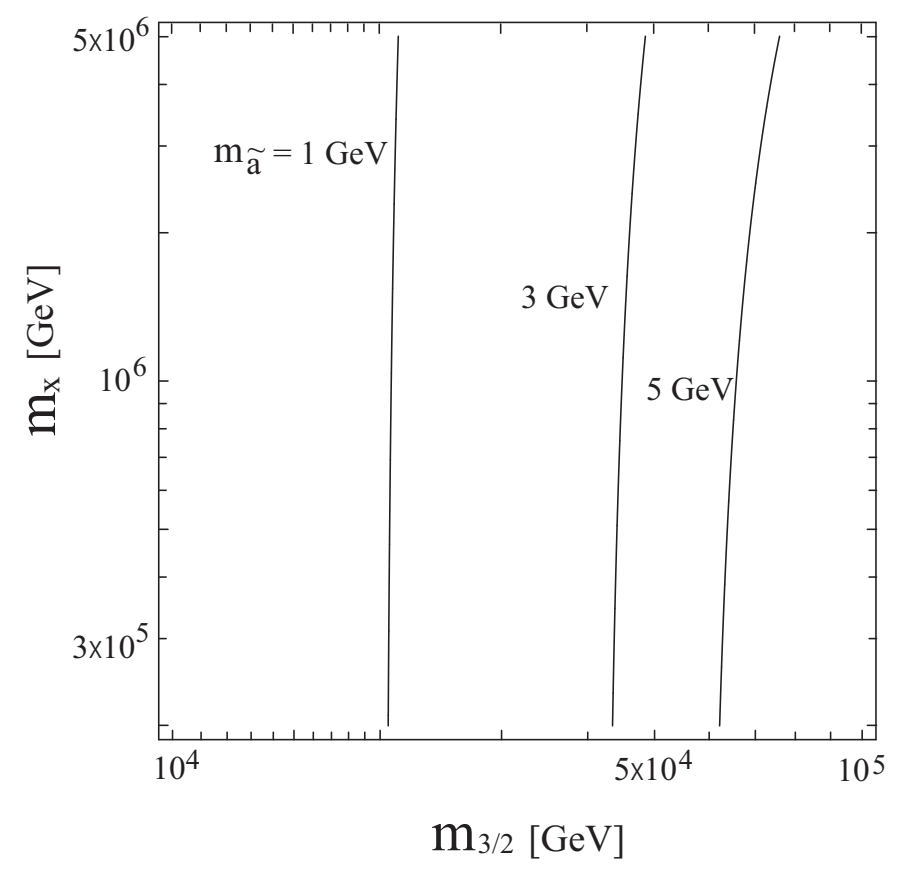

Figure 5: Contours of the axino mass, $m_{\widetilde{a}}$, which satisfy $\Omega_{\widetilde{a}} h^{2}=0.1$ drawn in $m_{3 / 2}-m_{X}$ plane in the case where NLSP is the wino with $m_{\widetilde{W}} \simeq 100 \mathrm{GeV}$ and their annihilation is effective. Three lines represent $m_{\widetilde{a}}=1,3$ and $5 \mathrm{GeV}$, from left to right. Here, we set $\lambda=N=1, k=0$ and $m_{\widetilde{W}}$ $=100 \mathrm{GeV}$ for simplicity. From the gravitino problem point of view, the gravitino mass should be heavier than about $30 \mathrm{TeV}$. 